\title{
CEM03 and LAQGSM03: Extension of the CEM2k+GEM2 and LAQGSM Codes to Describe Photo-Nuclear Reactions at Intermediate Energies (30 MeV to $1.5 \mathrm{GeV}$ )
}

\author{
S. G. Mashnik, ${ }^{*}$, a M. I. Baznat, ${ }^{\text {b K. K. Gudima, }}{ }^{\text {b }}$ A. J. Sierk, ${ }^{\text {a }}$ and R. E. Prael ${ }^{\text {a }}$ \\ ${ }^{a}$ Los Alamos National Laboratory, Los Alamos, New Mexico 87545, USA \\ ${ }^{\mathrm{b}}$ Institute of Applied Physics, Academy of Science of Moldova, Chisinau, Moldova
}

Received: April 30, 2005; In Final Form: July 26, 2005

\begin{abstract}
The improved Cascade-Exciton Model (CEM) code CEM2k+GEM2 and the Los Alamos version of the QuarkGluon String Model code LAQGSM are extended to describe photonuclear reactions. First, we incorporate new evaluations of elementary cross sections based on the latest experimental data into CEM2k+GEM2 and also make several improvements in the description of the de-excitation of nuclei remaining after the cascade stage of reactions induced by arbitrary projectiles. Next, for photonuclear reactions we include a normalization to evaluated experimental absorption cross sections based on the recent systematics by Kossov in CEM2k+GEM2. Then, we extend our high-energy code LAQGSM by adding the photonuclear mode which was ignored in all its previous versions, and add to it the photonuclear part from our improved CEM2k+GEM2. Presently, these codes do not include a calculation for GDR and describe properly photonuclear reactions only at intermediate energies from $\sim 30 \mathrm{MeV}$ to $\sim 1.5 \mathrm{GeV}$. In this work we present a short description of the photonuclear mode as incorporated into our codes, show several illustrative results, and point out some unresolved problems.
\end{abstract}

\section{Introduction}

The 2003 version $^{1-3}$ of the improved ${ }^{4}$ Cascade-Exciton Model $(\mathrm{CEM})^{5}$ as realized in the code CEM2k merged ${ }^{6-8}$ with the Generalized Evaporation Model code GEM2 by Furihata ${ }^{9}$ and of the Los Alamos version of the Quark-Gluon String Model code LAQGSM $^{10}$ merged $^{6-8}$ with GEM2 have been recently incorporated into the transport codes MARS $15^{11}$ and LAHET $3^{12}$ and are planned to be incorporated in the future into the transport codes MCNPX ${ }^{13}$ and MCNP6. ${ }^{14}$ Initially, neither CEM2k+ GEM2 nor LAQGSM+GEM2 considered photonuclear reactions and were not able to describe such reactions, either as stand-alone codes or as event generators in transport codes. To address this problem, here we extend CEM03 (the 2003 version of CEM $2 \mathrm{k}+\mathrm{GEM} 2$ ) and LAQGSM03 (the 2003 version of LAQGSM+GEM2) codes to describe photonuclear reactions at intermediate energies (from $\sim 30 \mathrm{MeV}$ to $\sim 1.5 \mathrm{GeV}$ ). We develop a model that is based on the Dubna IntraNuclear Cascade (INC) Photonuclear Reaction Model (PRM), ${ }^{15-17}$ uses experimental data now available in the literature, and a revision of recent systematics for the total photoabsorption cross sections by Kossov. ${ }^{18}$ Our photonuclear reaction model still has some problems and is under further development, but even the current version allows us to describe reasonably well intermediate energy photonuclear reactions. In the following, we present a description of our model together with several illustrative results.

\section{Dubna Photonuclear Reaction Mode}

The Dubna intranuclear cascade photonuclear reaction model (Dubna INC) was initially developed 35 years ago by one of us (KKG) in collaboration with Iljinov and Toneev ${ }^{15}$ to describe photonuclear reactions at energies above the Giant Dipole Resonance (GDR) region. [At photon energies $T_{\gamma}=$ $10-40 \mathrm{MeV}$, the de Broglie wavelength $\lambda$ is of the order of 20-5 fm, greater than the average inter-nucleonic distance in

*Corresponding author. E-mail: mashnik@lanl.gov. FAX: +1505-667-1931. the nucleus; the photons interact with the nuclear dipole resonance as a whole, thus the INC is not applicable.] Below the pion production threshold, the Dubna INC considers absorption of photons on only "quasi-deuteron" pairs according to the Levinger model: ${ }^{19}$

$$
\sigma_{\gamma A}=L \frac{Z(A-Z)}{A} \sigma_{\gamma \mathrm{d}},
$$

where $A$ and $Z$ are the mass and charge numbers of the nucleus, $L \approx 10$, and $\sigma_{\gamma \mathrm{d}}$ is the total photoabsorption cross section on deuterons as defined from experimental data.

At photon energies above the pion-production threshold, the Dubna INC considers production of one or two pions; the concrete mode of the reaction is chosen by the Monte Carlo method according to the partial cross sections (defined from available experimental data):

$$
\begin{aligned}
\gamma+\mathrm{p} & \rightarrow \mathrm{p}+\pi^{0}, \\
& \rightarrow \mathrm{n}+\pi^{+}, \\
& \rightarrow \mathrm{p}+\pi^{+}+\pi^{-}, \\
& \rightarrow \mathrm{p}+\pi^{0}+\pi^{0}, \\
& \rightarrow \mathrm{n}+\pi^{+}+\pi^{0} .
\end{aligned}
$$

The cross sections of $\gamma+\mathrm{n}$ interactions are derived from consideration of isotopic invariance, i.e. it is assumed that $\sigma(\gamma$ $+\mathrm{n})=\sigma(\gamma+\mathrm{p})$. The Compton effect on intranuclear nucleons is neglected, as its cross section is less than $\approx 2 \%$ of other reaction modes (see, e.g. Figure 6.13 in Reference 20). The Dubna INC does not consider processes involving production of three and more pions; this limits the model applicability to photon energies $T_{\gamma} \lesssim 1.5 \mathrm{GeV}$ [for $T_{\gamma}$ higher than the threshold for three-pion production, the sum of the cross sections (4)-(6) is assumed to be equal to the difference between the total inelastic $\gamma+p$ cross section and the sum of the cross sections of the twobody reactions (2) $-(3)]$.

The kinematics of two-body reactions (2)-(3) and absorption of photons by a pair of nucleons is completely defined by a given direction of emission of one of the secondary particles. Similarly to the procedure followed for $N+N$ and $\pi+N$ inter- 
actions, ${ }^{21,22}$ the cosine of the angle of emission of secondary particles can be represented in the center of mass (c.m.) system as a function of a random number $\xi$, distributed uniformly in the interval $[0,1]$

$$
\cos \theta=2 \xi^{1 / 2}\left[\sum_{n=0}^{N} a_{n} \xi^{n}+\left(1-\sum_{n=0}^{N} a_{n}\right) \xi^{N+1}\right]-1,
$$

where $N=M=3$,

$$
a_{n}=\sum_{k=0}^{M} a_{n k} T_{\gamma}^{k}
$$

where the coefficients $a_{n k}$ were fitted to describe the available experimental data and are published in Tables 1 and 2 of Reference 17 (the corresponding coefficients for $N+N$ and $\pi+$ $N$ interactions are published in Table 3 of Reference 22 and in Table 72 of the monograph by Barashenkov and Toneev ${ }^{16}$ ). The distribution of the secondary particles over the azimuthal angle $\varphi$ is assumed to be isotropic. After simulating the angle $\Theta_{1}$, using eqs 7 and 8 , and $\varphi_{1}$ isotropically for the first particle of any reaction with two particles in the final state, the angles $\Theta_{2}$ and $\varphi_{2}$ of the second particle, as well as the energies of both particles $T_{1}$ and $T_{2}$ are uniquely determined from fourmomentum conservation.

The analysis of experimental data has shown that the channel (4) of two-pion photoproduction proceeds mainly through the decay of the $\Delta^{++}$isobar listed in the last Review of Particle Physics by the Particle Data Group ${ }^{23}$ as having the mass $M=1232 \mathrm{MeV}$

$$
\begin{aligned}
\gamma+\mathrm{p} & \rightarrow \Delta^{++}+\pi^{-} \\
\Delta^{++} & \rightarrow \mathrm{p}+\pi^{+},
\end{aligned}
$$

whereas the production cross section of other isobar components $\left(\frac{3}{2}, \frac{3}{2}\right)$ are small and can be neglected. The Dubna INC uses the Lindenbaum-Sternheimer resonance model ${ }^{24}$ to simulate the reaction (9). In accordance with this model, the mass of the isobar $M$ is determined from the distribution

$$
\frac{\mathrm{d} W}{\mathrm{~d} M} \sim F(E, M) \sigma(M),
$$

where $E$ is the total energy of the system, $F$ is the two-body phase space of the isobar and $\pi^{-}$meson, and $\sigma$ is the isobar production cross section which is assumed to be equal to the cross section for elastic $\pi^{+} p$ scattering.

The c.m. emission angle of the isobar is approximated using eqs 7 and 8 with the coefficients $a_{n k}$ listed in Table 3 of Reference 17; isotropy of the decay of the isobar in its c.m. system is assumed.

In order to calculate the kinematics of the non-resonant part of the reaction (4) and two remaining three-body channels (5) and (6), the Dubna INC uses the statistical model. The total energies of the two particles (pions) in the c.m. system are determined from the distribution

$$
\frac{\mathrm{d} W}{\mathrm{~d} E_{\pi_{1}} \mathrm{~d} E_{\pi_{2}}} \sim\left(E-E_{\pi_{1}}-E_{\pi_{2}}\right) E_{\pi_{1}} E_{\pi_{2}} / E,
$$

and that of the third particle (nucleon, $N$ ) from conservation of energy. The actual simulation of such reactions is done as follows: Using a random number $\xi$, we simulate in the beginning the energy of the first pion using

$$
E_{\pi_{1}}=m_{\pi_{1}}+\xi\left(E_{\pi_{1}}^{\max }-m_{\pi_{1}}\right)
$$

where

$$
E_{\pi_{1}}^{\max }=\left[E^{2}+m_{\pi_{1}}^{2}-\left(m_{\pi_{2}}+m_{N}\right)^{2}\right] / 2 E .
$$

Then, we simulate the energy of the second pion $E_{\pi_{2}}$ according to eq 11 using the Monte Carlo rejection method. The energy of the nucleon is then calculated as $E_{N}=E-E_{\pi_{1}}-E_{\pi_{2}}$, checking that the "triangle law" for momenta

$$
\left|p_{\pi_{1}}-p_{\pi_{2}}\right| \leq p_{N} \leq\left|p_{\pi_{1}}+p_{\pi_{2}}\right|
$$

is fulfilled, otherwise this sampling is rejected and the procedure is repeated. The angles $\Theta$ and $\varphi$ of the pions are sampled assuming an isotropic distribution of particles in the c.m. system,

$$
\begin{aligned}
\cos \Theta_{\pi_{1}} & =2 \xi_{1}-1, & \cos \Theta_{\pi_{2}} & =2 \xi_{2}-1, \\
\varphi_{\pi_{1}} & =2 \pi \xi_{3}, & \varphi_{\pi_{2}} & =2 \pi \xi_{4},
\end{aligned}
$$

and the angles of the nucleon are defined from momentum conservation, $\vec{p}_{N}=-\left(\vec{p}_{\pi_{1}}+\vec{p}_{\pi_{2}}\right)$.

So an interaction of a photon with a nucleon inside a nucleus leads to two or three fast cascade particles. Depending on their momenta and coordinates, these particles can leave the nucleus, be absorbed, or initiate a further intranuclear cascade. All the remaining details of the Dubna INC (followed by the evaporation/fission of excited nuclei produced after the cascade stage of reactions) calculation are the same as for $N+A$ and $\pi+A$ reactions and are described in detail in the monograph. ${ }^{16}$

The Dubna INC PRM was used successfully for many years as a stand-alone model to study different aspects of photonuclear reactions and was also incorporated without modifications into the transport codes CASCADE ${ }^{25}$ and GEANT4, ${ }^{26}$ and with some improvements, via CEM95, ${ }^{27} \mathrm{CEM} 97,{ }^{28}$ and CEM2k, ${ }^{4}$ into the transport codes MARS14 ${ }^{29}$ and MCNPX, ${ }^{13,}{ }^{30,31}$ respectively. In the middle of the $1970 \mathrm{~s}$, one of the authors of the initial version of the Dubna INC PRM, Iljinov, moved from JINR, Dubna to INR, Moscow and continued to develop further the Dubna INC with his Moscow Group, which evolved into what is now known in the literature as the Moscow INC model (see, e.g. Reference 32 and references therein). The Moscow INC model was recently extended to describe photonuclear reactions at energies up to $10 \mathrm{GeV}^{33}$

\section{From CEM95 to CEM03}

Photonuclear reactions were not considered in the initial version of the CEM. ${ }^{5}$ The Dubna PRM was incorporated ${ }^{34}$ first into the CEM95 ${ }^{27}$ version of the CEM and used thereafter to analyze a large number of photonuclear reactions. ${ }^{35}$ Later on, CEM95 was incorporated as an event generator into the MARS $14^{29}$ transport code and used in some applications.

By early 1997 , one of the authors of the CEM (SGM) moved from JINR, Dubna to LANL, Los Alamos, and continued to develop further with his collaborators the cascade-exciton model for LANL needs, e.g. as an event generator for the Los Alamos transport code $\mathrm{MCNPX}^{13}$ and for other applications.

3.1. New Approximations for $\gamma p$ Cross Sections. The first improvements in the CEM of the photonuclear mode of the Dubna INC was done in the CEM97 version ${ }^{28}$ of the CEM. The improved cascade-exciton model in the code CEM97 differs from the older CEM95 version by incorporating new approximations for the elementary $N N, \pi N$, and $\gamma_{\mathrm{p}}$ cross sections used in the cascade, using more precise values for nuclear masses and pairing energies, employing a corrected systematics for the level-density parameters, adjusting the cross sections for pion absorption on quasi-deuteron pairs inside a nucleus, including the Pauli principle in the preequilibrium calculation, and improving the calculation of fission widths. Implementation of significant refinements and improvements in the algorithms of many subroutines led to a decrease of the computing time by up to a factor of 6 for heavy nuclei, which is very important when performing simulations with transport codes.

Concerning specifically the photonuclear reactions, in CEM97 we developed improved approximations for the elementary $\gamma_{\mathrm{p}}$ cross sections compared with the Dubna INC PRM. ${ }^{15}$ 
In the Dubna INC PRM ${ }^{15}$ used in CEM95, the cross sections for the free $\gamma \mathrm{p}$ (and for $N N$ and $\pi N$ ) interactions are approximated using a special algorithm of interpolation/extrapolation through a number of picked points, mapping the experimental data as possible. This was done very accurately by the authors of the Dubna INC PRM ${ }^{15}$ using all experimental data available at that time, about 35 years ago. Currently there are many more experimental data on cross section; therefore we revised the approximations of all elementary cross sections used in CEM97. ${ }^{28}$ We collected all published experimental data from available sources, then developed an improved algorithm for approximating cross sections and developed simple and fast approximations for elementary cross sections which fit very well presently available experimental data not only up to $\sim 1.5$ $\mathrm{GeV}$, where the Dubna INC PRM is assumed to be used, or up to about $5 \mathrm{GeV}$, the upper recommended energy for the present version of the CEM for nucleon- and pion-induced reactions, but up to 50-100 GeV and higher, depending on availability of data. So far we have such approximations for 8 different
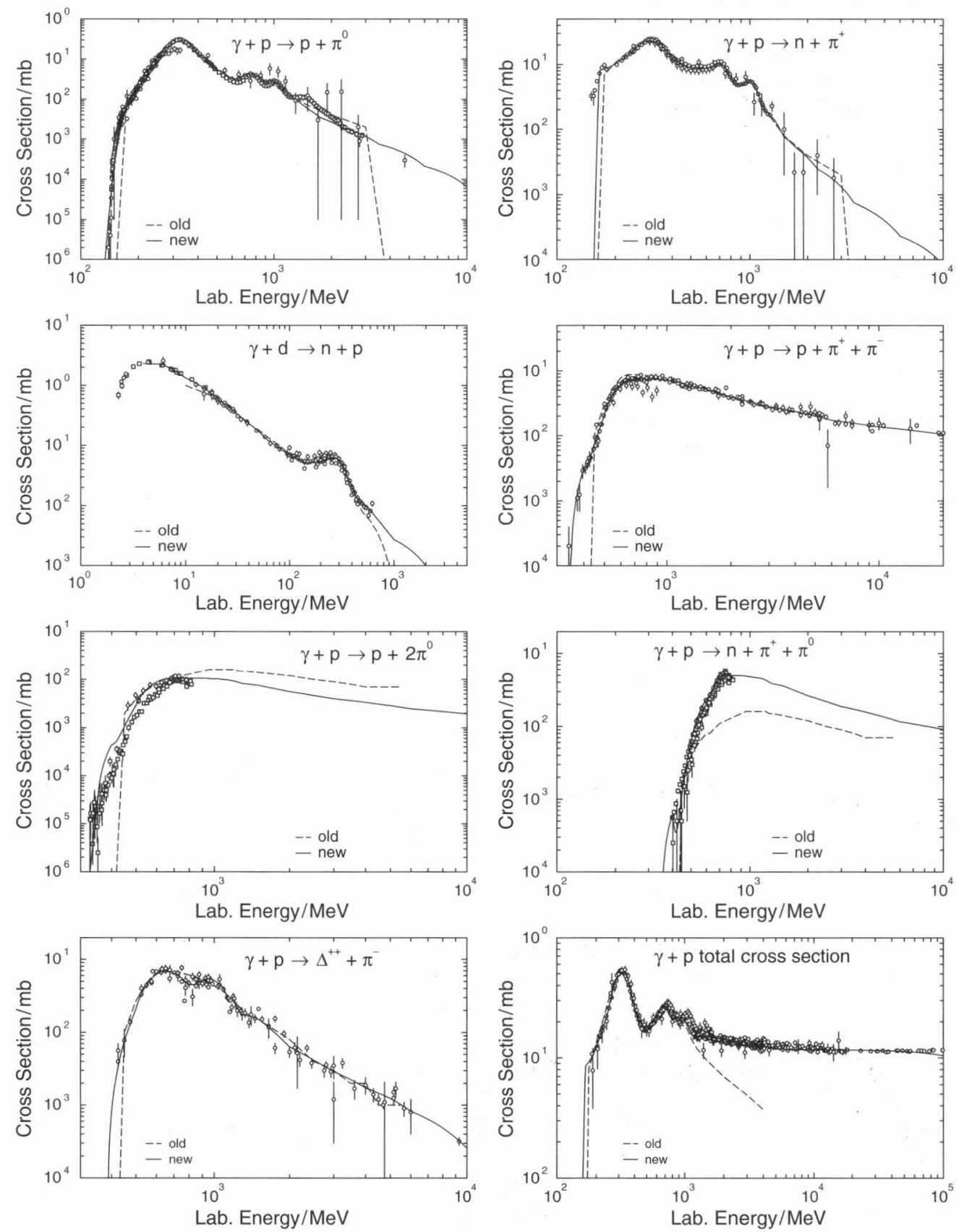

Figure 1. Comparison of eight experimental total $\gamma+p(d)$ cross sections with the old approximations from the Dubna INC PRM and with our new approximations incorporated into the CEM03 and LAQGSM03 codes. Experimental data (circles and squares) are compiled from: $\gamma+\mathrm{p} \rightarrow \mathrm{p}+\pi^{0}$ : Refs. 36-45; $\gamma+\mathrm{p} \rightarrow \mathrm{n}+\pi^{+}$: Refs. 36-39 and 46-51; $\gamma+\mathrm{d} \rightarrow \mathrm{n}+\mathrm{p}$ : Refs. 23, 37, and 52-72; $\gamma+\mathrm{p} \rightarrow \mathrm{p}+\pi^{+}+\pi^{-}:$Refs. 37, 42, and 73-76; $\gamma+\mathrm{p}$ $\rightarrow \mathrm{p}+2 \pi^{0}:$ Refs. 73 and $77-79 ; \gamma+\mathrm{p} \rightarrow \mathrm{n}+\pi^{+}+\pi^{0}$ : Refs. 73,80 , and $81 ; \gamma+\mathrm{p} \rightarrow \Delta^{++}+\pi^{-}$: Ref. 37; $\gamma+$ p total cross sections: Refs. $23,37,51$, and 52 , respectively. The squares show recent experimental data that became available to us after we completed our fit; although these recent data agree reasonably well with our approximations, a re-fitting would slightly improve the agreement. 
data than 35 years ago when the Dubna group produced their approximations used in the Dubna INC PRM,${ }^{15}$ for a number of interaction modes like the total $\gamma p$ cross sections at energies below $1.2 \mathrm{GeV}$ (where the initial Dubna INC PRM was assumed to be used), and for such modes as $\gamma+p \rightarrow p+\pi^{0}, \gamma$ $+\mathrm{p} \rightarrow \mathrm{n}+\pi^{+}, \gamma+\mathrm{p} \rightarrow \mathrm{p}+\pi^{+}+\pi^{-}$, and $\gamma+\mathrm{d} \rightarrow \mathrm{n}+\mathrm{p}$, at energies not too close to their thresholds, the original approximations also agree very well with presently available data, in the energy region where the Dubna INC PRM was developed to work. This is a partial explanation of why the old Dubna $\mathrm{INC}^{16}$ and the younger CEM95 ${ }^{27}$ describe so well many characteristics of different nuclear reactions. On the other hand, for some elementary cross sections like $\gamma+p \rightarrow p+2 \pi^{0}$ and $\gamma$ $+\mathrm{p} \rightarrow \mathrm{n}+\pi^{+}+\pi^{0}$, the old approximations differ significantly from the present data, demonstrating the need for a better description of all modes of photonuclear reactions. (Similar results were obtained in CEM $97^{28}$ for hadron-hadron cross section approximations.)

The CEM97 code with these cross sections and the other mentioned improvements was incorporated by Gallmeier ${ }^{30}$ into the MCNPX transport code, ${ }^{13}$ allowing MCNPX to consider for the first time interaction of intermediate-energy photons with thick targets of practically arbitrary geometry.

3.2. New Approximations for Differential $\gamma+p$ Cross Sections. The CEM $2 \mathrm{k}^{4}$ version of CEM is a "new generation" of the CEM following CEM97. ${ }^{28}$ Its development was partially motivated by the availability of some new, very precise and useful experimental data obtained recently at GSI in Darmstadt, Germany, where a large number of measurements have been performed using inverse kinematics for interactions of ${ }^{56} \mathrm{Fe}$, ${ }^{197} \mathrm{Au},{ }^{208} \mathrm{~Pb}$, and ${ }^{238} \mathrm{U}$ at $1500,1000,800,750,500$, and 300 $\mathrm{MeV} /$ nucleon with liquid ${ }^{1} \mathrm{H}$. These measurements provide a large set of cross sections for production of practically all possible isotopes from such reactions in a "pure" form, i.e. individual cross sections from a specific given bombarding isotope (or target isotope, when considering reactions in the usual kinematics, $\mathrm{p}+\mathrm{A}$ ). Such cross sections are much easier to compare to models than the "camouflaged" data from $\gamma$ spectrometry measurements. In addition, many reactions where a beam of light, medium, or heavy ions with energy near to or below $1 \mathrm{GeV} /$ nucleon interact with different nuclei, from the lightest, $\mathrm{d}$, to the heaviest, ${ }^{208} \mathrm{~Pb}$, were measured recently at GSI. References on these measurements and many tabulated experimental cross sections may be found on the Web page of Schmidt. ${ }^{82}$

(We have analyzed with CEM2k and LAQGSM all measurements done at GSI of which we are aware, both for protonnucleus and nucleus-nucleus interactions; some examples of our results compared with the GSI data and calculations by other available models may be found in Reference 3 and references therein.)

During the development of the CEM2k version of CEM and
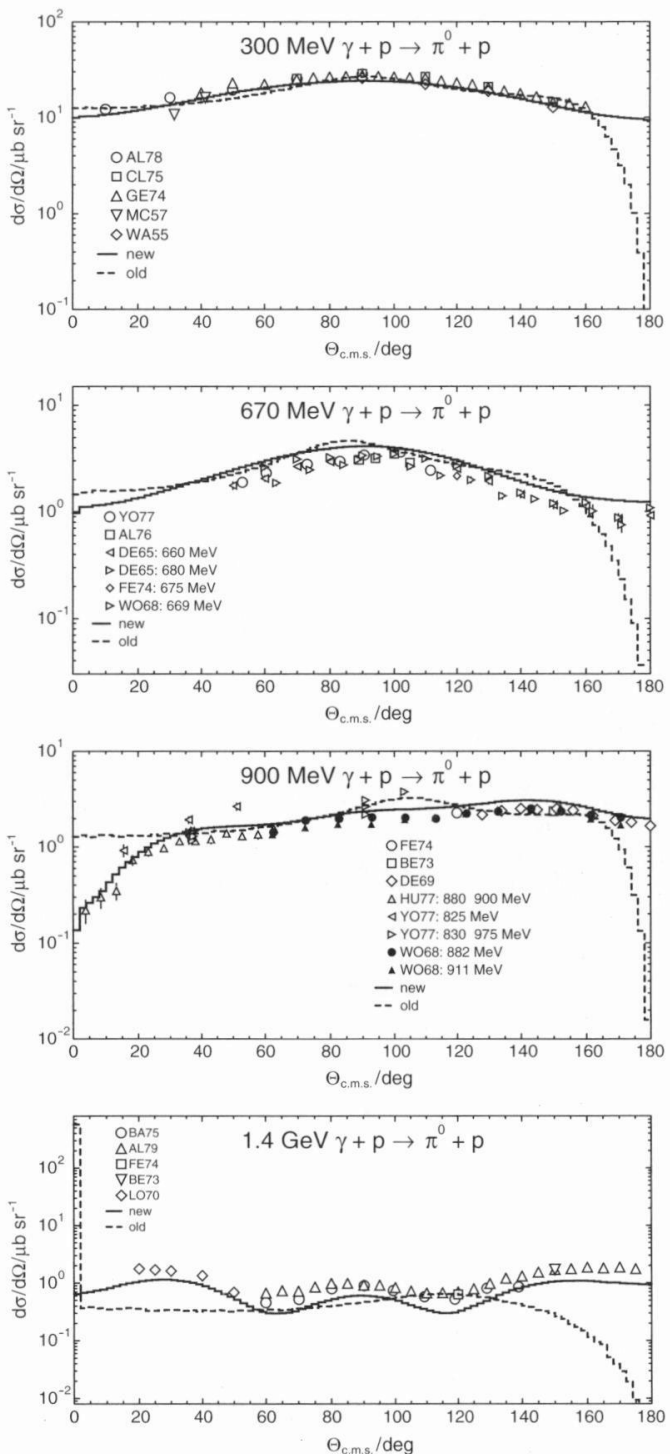

Figure 2. Example of eight angular distributions of $\pi^{0}$ from $\gamma \mathrm{p} \rightarrow \pi^{0} \mathrm{p}$ as functions of $\Theta_{\text {c.m.s. }}^{\pi}$ at photon energies from $260 \mathrm{MeV}$ to $1.4 \mathrm{GeV}$. The dashed lines show the old approximations of the Dubna INC PRM while the solid lines are our new approximations incorporated into the CEM03 and LAQGSM03 codes. Experimental data are shown by symbols and are from: CL75, ${ }^{83}$ GE74 ${ }^{84}$ FI70 ${ }^{85}$ MC57 ${ }^{86}$ AL78 ${ }^{87}$ WA55, ${ }^{88}$ YO $77,{ }^{89}$ BE73 ${ }^{90}$ AL76,${ }^{91}$ DE65,${ }^{92}$ FE74, ${ }^{93}$ WO68,${ }^{94}$ WE78, ${ }^{95}$ DE69,${ }^{96}$ WO60,${ }^{97}$ HU77,${ }^{98}$ BA75, ${ }^{99}$ JA60,${ }^{100}$ AL79,,${ }^{101}$ and LO70 $;{ }^{102}$ tabulated values are available at: http://wwwspires. dur.ac.uk/ hepdata/reac2.html. 
of LAQGSM, we concentrated mainly on proton-nucleus and nucleus-nucleus reactions and tried to improve the general description of different types of nuclear reactions by our models, without focusing specifically on photonuclear reactions. The main difference of CEM $2 \mathrm{k}$ from its precursor CEM97 is in the criterion for when to move from the intranuclear-cascade stage of a reaction to its preequilibrium stage, and when to move from the latter to the evaporation/fission slow stage of the reaction. In short, CEM2k has a longer cascade stage, less preequilibrium emission, and a longer evaporation stage with a higher excitation energy, as compared to CEM97 and CEM95. Besides these changes to CEM97, we also made in CEM2k a number of other improvements and refinements, such as imposing momentumenergy conservation for each simulated event (the Monte Carlo algorithm previously used in CEM provides momentum-energy conservation only statistically, on the average, but not exactly for each simulated event); using real binding energies for nucleons at the cascade stage of a reaction instead of the approximation of a constant separation energy of $7 \mathrm{MeV}$ used in the previous versions of the CEM; using reduced masses of particles in the calculation of their emission widths instead of using the approximation of no recoil used in the previous versions; and coalescence of complex particles from fast cascade nucleons already outside the nucleus. On the whole, CEM2k describes many nuclear reactions, including the ones induced by photons better than CEM97 and CEM95 do. CEM2k was incorporated by Gallmeier into MCNPX to replace CEM97, and this version of MCNPX was extended by him to describe photonuclear reactions also in the GDR region ${ }^{31}$ (as a stand-alone code, CEM2k was developed to describe photonuclear reactions only at energies above the GDR region).

We have focused on the improved description of specifically the photonuclear reactions when developing our latest version of CEM, CEM03. ${ }^{1}$ Although CEM97 contained new approximations and algorithms to better describe the integrated elementary $N N, \pi N$, and $\gamma N$ cross sections as mentioned above, the double differential distributions of secondary particles from such interactions were simulated by CEM $2 \mathrm{k}$ and all its precursors using the old Dubna INC approximations (7)-(11) for $\gamma \mathrm{p}$ reactions (and similar relations, for $N N$ and $\pi N$ collisions). These were obtained by Gudima et al. ${ }^{15,21,22} 36$ years ago, using the measurements available at that time. In CEM03, for $N N$ and $\pi N$ collisions, we addressed this problem by developing new approximations similar to (7)-(11) and by using recent systematics by other authors, based on the experimental data available today (see details on $N N$ and $\pi N$ reactions in Reference 1). In the case of $\gamma \mathrm{p}$ reactions (2) and (3), we chose another way: Instead of fitting the parameters $a_{n}$ from eq 7 at different $E_{\gamma}$ we found data (see, e.g. Figures 2 and 3 ) and finding the energy dependence of parameters $a_{n k}$ in eq 8 using the values obtained for $a_{n}$ as was done in the old Dubna INC, we took advantage of the event generator for $\gamma \mathrm{p}$ and $\gamma \mathrm{n}$ reactions from the Moscow
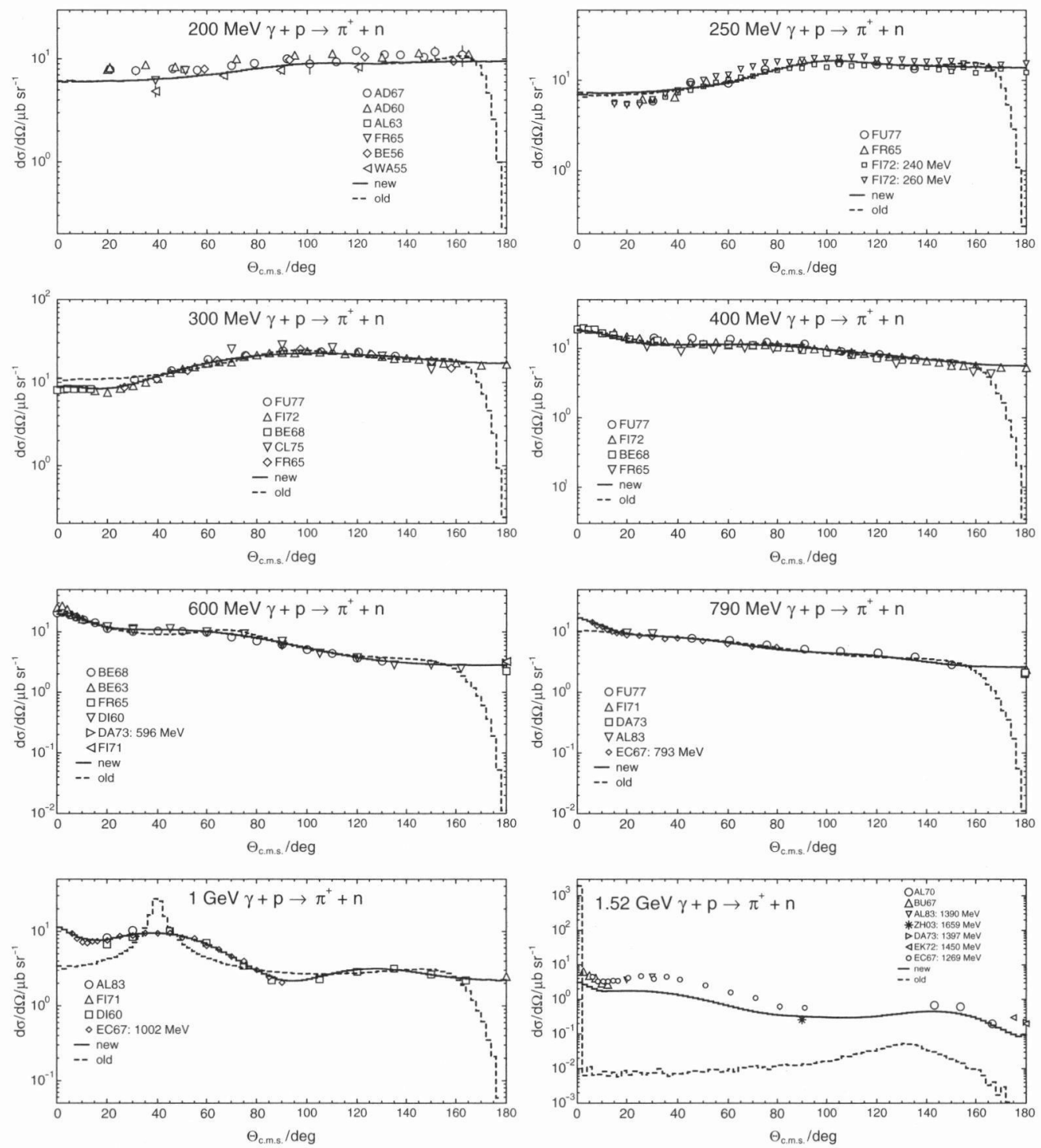

Figure 3. Example of eight angular distributions of $\pi^{+}$from $\gamma p \rightarrow \pi^{+} \mathrm{n}$ as functions of $\Theta_{\mathrm{c} . \mathrm{m} . \mathrm{s}}^{\pi}$ at photon energies from $200 \mathrm{MeV}$ to $1.52 \mathrm{GeV}$. The dashed lines show the old approximations of the Dubna INC PRM while the solid lines are our new approximations incorporated into the CEM03 and LAQGSM03 codes. Experimental data are shown by symbols and are from: AD67, ${ }^{103}$ AD60, ${ }^{104}$ AL63, ${ }^{105}$ FR65, ${ }^{106}$ BE56, ${ }^{107}$ WA55, ${ }^{108}$ FU77, ${ }^{109}$ FI72, ${ }^{110}$ BE68, ${ }^{111}$ CL75, ${ }^{83}$ BE63, ${ }^{112}$ DI60,${ }^{113}$ DA73, ${ }^{114}$ FI71, ${ }^{115}$ AL83, ${ }^{116}$ EC67, ${ }^{117}$ AL70, ${ }^{118}$ BU67, ${ }^{119}$ ZH03, ${ }^{120}$ and EK72; ${ }^{121}$ tabulated values are available at: http://wwwspires.dur.ac.uk/ hepdata/reac2.html. 
$\mathrm{INC}^{33}$ kindly sent us by Pshenichnov. That event generator includes'a data file with smooth approximations through presently available experimental data at 50 different gamma energies from 117.65 to $6054 \mathrm{MeV}$ (in the system where the p or $n$ interacting with $\gamma$ is at rest) for the c.m. angular distributions $\mathrm{d} \sigma / \mathrm{d} \Omega$ of secondary particles as functions of $\Theta$ tabulated for values of $\Theta$ from 0 to $180^{\circ}$ with the step $\Delta \Theta=10^{\circ}$ for 60 different channels of $\gamma \mathrm{p}$ and $\gamma \mathrm{n}$ reactions considered by the Moscow INC (see details in Reference 33). We use part of that data file with data for reactions (2) and (3), and have written an algorithm to simulate unambiguously $\mathrm{d} \sigma / \mathrm{d} \Omega$ and to choose the corresponding value of $\Theta$ for any $E_{\gamma}$, using a single random number $\xi$ uniformly distributed in the interval $[0,1]$. This is straightforward due to the fact that the function $\xi(\cos \Theta)$

$$
\xi(\cos \Theta)=\int_{-1}^{\cos \Theta} \mathrm{d} \sigma / \mathrm{d} \Omega \mathrm{d} \cos \Theta / \int_{-1}^{1} \mathrm{~d} \sigma / \mathrm{d} \Omega \mathrm{d} \cos \Theta
$$

is a smooth monotonic function increasing from 0 to 1 as cos $\Theta$ varies from -1 to 1 . Naturally, when $E_{\gamma}$ differs from the values tabulated in the data file, we perform first the needed interpolation in energy. We use this procedure to describe angular distributions of secondary particles from reactions (2) and (3), as well as for isotopically symmetric reactions $\gamma+\mathrm{n}$ $\rightarrow \mathrm{n}+\pi^{0}$ and $\gamma+\mathrm{n} \rightarrow \mathrm{p}+\pi^{-}$in CEM03.

Examples of eight angular distributions of $\pi^{0}$ from $\gamma \mathrm{p} \rightarrow \pi^{0} \mathrm{p}$ and of $\pi^{+}$from $\gamma p \rightarrow \pi^{+} n$ as functions of $\Theta_{\text {c.m.s }}^{\pi}$ are presented in Figures 2 and 3 . We see that the approximations developed in CEM03 (solid histograms) agree much better with the available experimental data than the old Dubna INC approximations (7) $-(8)$ used in all precursors of CEM03 (dashed histograms).

\section{New Approximations for $\gamma+A$ Absorption Cross Sections}

CEM03 (and its predecessors) does not consider absorption of low energy photons in the GDR region and takes into account photoproduction on free nucleons of only two pions. This restricts its applicability to the range $30 \mathrm{MeV} \lesssim E_{\gamma} \lesssim 1.5$ $\mathrm{GeV}$, which is not convenient when it is used as an event generator in a transport code.

To extend the applicability of CEM03 (and LAQGSM03) into the GDR region, it is necessary to omit the intranuclear cascade (INC) and to consider such reactions as starting with the preequilibrium model. The INC used by CEM03 as the first stage of arbitrary reactions is a semiclassical model that does not consider any collective degrees of freedom of a nucleus, including the GDR; in addition, the photon-energy in the GDR region is too low to justify the use of any INC. In our approach, it is possible to deal with this limitation as was done 30 years ago, ${ }^{122}$ using the Modified Exciton Model (MEM) ${ }^{123,124}$ used in the initial version of $\mathrm{CEM}^{5}$ and 25 years later, ${ }^{125}$ using an improved version of the MEM contained in the CEM $95^{27}$ code. We plan to extend CEM03 to describe photonuclear reactions in the GDR region in the near future, but this requires a large amount of tedious work: 1) to make sure that we use the most reliable parameters of the GDR for all nuclei, 2) to define an optimal transition from the current three-stage (INC, preequilibrium, and evaporation/fission) description of reactions to a two-stage approach needed in the GDR region, and 3) to test the extended model against the available experimental data.

To describe properly with CEM03 and LAQGSM03 photonuclear reactions above $E_{\gamma} \sim 1.5 \mathrm{GeV}$, it is necessary to take into account production of more than two pions in $\gamma N$ collisions, as well as to consider production of resonances heavier than $\Delta(1232)$, as has been done, i.e. in the Moscow INC. ${ }^{33} \mathrm{We}$ plan to extend CEM03 and LAQGSM03 to higher $E_{\gamma}$ in subsequent versions.

In the meantime, it is possible to get quite reasonable results for spectra of emitted nucleons and complex particles and for the nuclide production cross sections with our present CEM03 model for photonuclear reactions both in the GDR region and at $E_{\gamma} \gtrsim 1.5 \mathrm{GeV}$, by employing a correct total photoabsorption cross section. Indeed, CEM03 starts a reaction in the GDR region with a cascade and since the $\gamma$ energy is below the pionproduction threshold, the only available reaction channel is to absorb such photons on a quasi-deuteron pair of nucleons, generating two "cascade" nucleons inside the nucleus. As the energy of these nucleons is low, $\sim 10 \mathrm{MeV}$, these nucleons are "absorbed" by the nucleus generating two excited nucleons (excitons) and two holes, then CEM03 would proceed with this process as a preequilibrium reaction followed by evaporation/fission. All the real calculation of such reactions would be done with only the preequilibrium and evaporation models and the INC would serve only to provide the number of excitons, as an input to the MEM. At the end of the calculation, the total photoabsorption (reaction) cross section is needed to normalize the results. CEM03 (and most other INC models) calculates the total reaction cross section, $\sigma_{\text {in }}$, by the Monte Carlo method using the geometrical cross section, $\sigma_{\text {geom }}$, and the number of inelastic, $N_{\text {in }}$, and elastic, $N_{\text {el }}$, simulated events, namely: $\sigma_{\text {in }}=$ $\sigma_{\text {geom }} N_{\text {in }} /\left(N_{\text {in }}+N_{\text {el }}\right)$. This approach provides a good agreement with available data for reactions induced by nucleons, pions, and photons at incident energies above about $100 \mathrm{MeV}$, but is not reliable at energies below $100 \mathrm{MeV}$ (see, e.g. Figures 3 and 4 and Reference 7).

To address this problem for photonuclear reactions, we have written a FORTRAN routine called GABS.FOR based on the recent approximation by Kossov, ${ }^{18}$ that provides reliable photoabsorption cross sections on arbitrary targets at all energies from the hadron production threshold to about $40 \mathrm{TeV}$. We have added GABS.FOR to CEM03 to normalize our photonuclear results to this systematics rather than to $\sigma_{\text {in }}$ calculated by the Monte Carlo method, as we have done previously. (As a rule, we use LAQGSM03 only at energies above several GeV, where CEM03 becomes already not reliable; at such high energies, LAQGSM03 describes quite well $\sigma_{\text {in }}$ and does not require renormalization of its results to any systematics; therefore we do not incorporate GABS.FOR into LAQGSM03.)

The Kossov approximation ${ }^{18}$ of the energy dependence of photonuclear cross sections is subdivided into three main regions: the GDR region, the nucleon resonance region, and the high-energy region. Its functional form is also subdivided into three groups depending on the mass number of the target: the $\sigma_{\gamma \mathrm{p}}$ cross section, the cross section for $\gamma \mathrm{d}$ reactions, and the $\sigma_{\gamma A}$ cross section for $A>2$.

We note that eq 41 in Reference 18 was misprinted in the original publication by Kossov, where a "+" sign occurs instead of a "-" sign. The misprinted formula does not reproduce the cross sections presented in Reference 18, whereas the corrected version does.

Figures 4 and 5 show examples of twelve photoabsorption cross sections on several light, medium, and heavy nuclei. In these figures, we compare predictions of the Kossov systematics as implemented in the routine GABS.FOR with available experimental data and with the Los Alamos National Laboratory (LANL), Korean Atomic Energy Institute (KAERI), and the Institute of Physics and Power Engineering (IPPE), Obninsk, Russia and the Center for Photonuclear Experimental Data [Centr Dannykh Fotoyasdernykh Eksprtimentov (CDFE), in Russian], Moscow, Russia, Modified (MOD) Library of Photonuclear Data [Biblioteka Fotoyadernykh Dannykh (BOFOD), in Russian], BOFOD(MOD), evaluations from the IAEA Photonuclear Data Library, ${ }^{126}$ as well as with calculations by two older versions of CEM, namely, the CEM95 photonuclear code version $^{34}$ and CEM2k as modified by Gallmeier ${ }^{31}$ for MCNPX.

The Kossov systematics describe well the experimental photoabsorption cross sections and agree with the LANL, 

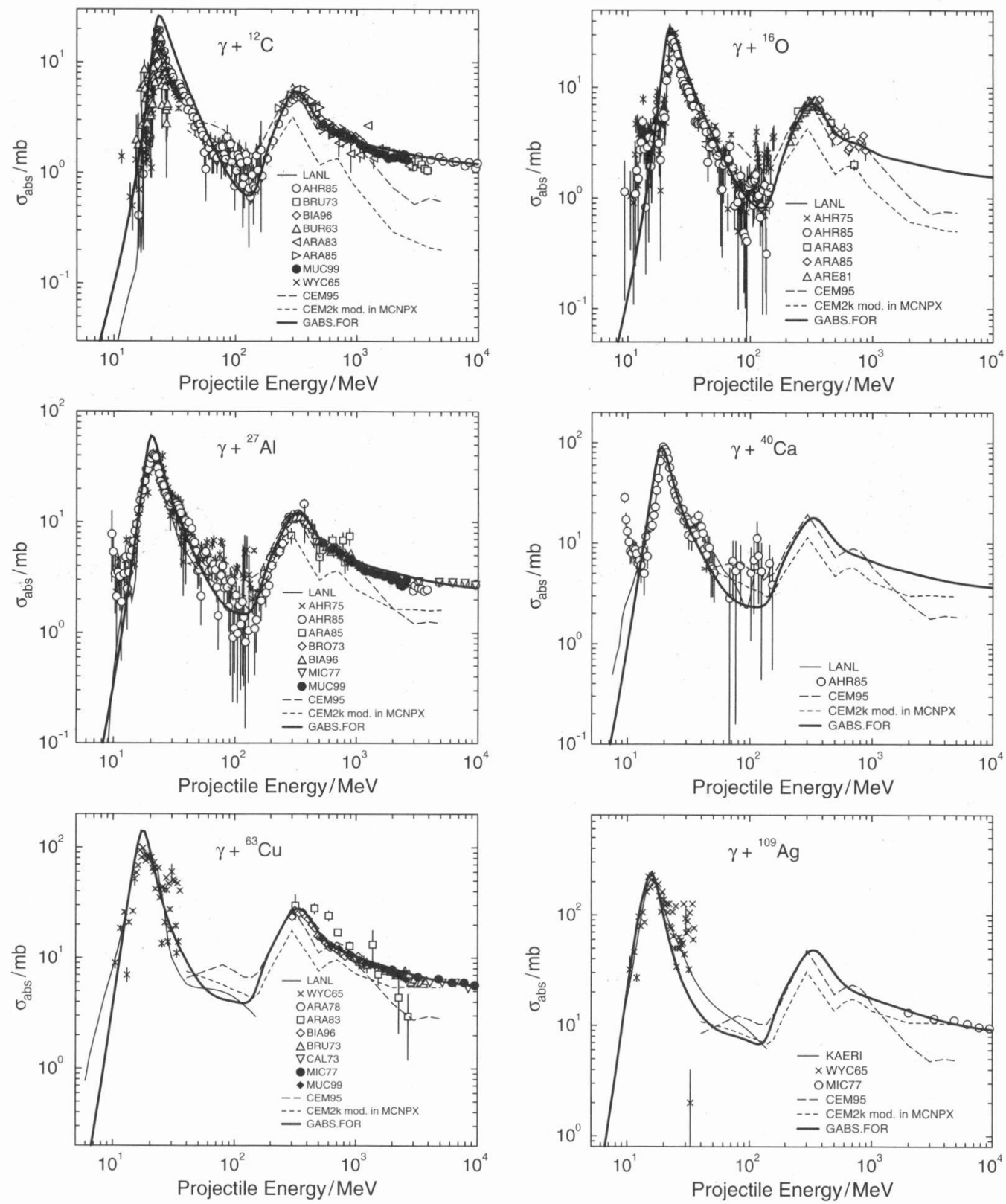

Figure 4. Examples of total photoabsorption cross sections for ${ }^{12} \mathrm{C},{ }^{16} \mathrm{O},{ }^{27} \mathrm{Al},{ }^{40} \mathrm{Ca},{ }^{63} \mathrm{Cu}$, and ${ }^{109} \mathrm{Ag}$ as functions of photon energy. The thick solid lines marked as "GABS.FOR" are from our subroutine written to reproduce Kossov's ${ }^{18}$ systematics, as described in the text. The thin solid line marked as "LANL" (or "KAERI", for ${ }^{109} \mathrm{Ag}$ ) show the evaluations by LANL (or KAERI, for ${ }^{109} \mathrm{Ag}$ ) from the IAEA Photonuclear Data Library. ${ }^{126}$ Results from the photonuclear version of CEM95 $5^{34}$ and from CEM2k as modified for MCNPX by Gallmeier ${ }^{31}$ are shown by the long-dashed and dashed lines, respectively. Experimental data (symbols) are from: AHR85, ${ }^{127}$ BRU73, ${ }^{128}$ BIA96, ${ }^{129}$ BUR63, ${ }^{130}$ ARA83, ${ }^{131}$ ARA85, ${ }^{132}$ MUC99, ${ }^{133}$ WYC65,${ }^{134}$ AHR75, ${ }^{135}$ ARE81, ${ }^{136}$ MIC77, ${ }^{137}$ ARA78, ${ }^{138}$ and CAL73. ${ }^{139}$

KAERI, and the BOFOD evaluations, especially for heavy targets. For ${ }^{12} \mathrm{C},{ }^{27} \mathrm{Al}$, and ${ }^{63} \mathrm{Cu}$ (and several other nuclei we tested but did not include in Figures 4 and 5) the agreement in the GDR region is not so good. This is because we use here the "global" approximation given by the Kossov systematics to calculate the photoabsorption cross section in the GDR region for all nuclei. It is known from the literature that the GDR of light nuclei differ significantly from the ones of heavy nuclei, and should be addressed carefully for each light nucleus separately. In fact, Kossov ${ }^{18}$ had fitted the parameters of the light nuclei separately and his results shown in Figures 2 to 7 of Reference 18 for the light nuclei agree better with the data than the "global" systematics shown here does. Unfortunately, Kossov did not publish the parameterization of the GDR he found for every light nucleus in Reference 18 (some details of this are listed in the recent GEANT4 Physics Reference Manual ${ }^{156}$ and in Reference 157, but only for some light nuclei, and those details differ from what is published in Reference 18). To fill this gap, we hope to determine ourselves a parameterization of the GDR photoabsorption on light nuclei using all available experimental data.

\section{Illustrative Results}

In this Section, we present several illustrative results from CEM03 and LAQGSM03 extended to describe photonuclear reactions. Let us start with photofission cross sections. We have compiled from the literature all reliable experimental data on photofission cross sections that we could identify and have analyzed them with both CEM03 and LAQGSM03. Figure 6 shows a comparison of such data for ${ }^{197} \mathrm{Au},{ }^{208} \mathrm{~Pb},{ }^{209} \mathrm{Bi},{ }^{232} \mathrm{Th}$, ${ }^{233} \mathrm{U},{ }^{235} \mathrm{U},{ }^{238} \mathrm{U}$, and ${ }^{237} \mathrm{~Np}$ with the results of CEM03, as well as to several earlier versions, namely, the photonuclear versions of CEM 95, ${ }^{34}$ CEM98, ${ }^{158}$ CEM $2 \mathrm{k}+\mathrm{GEM} 2,{ }^{8}$ and by the modified version of CEM $2 \mathrm{k}$ incorporated into MCNPX by Gallmeier. ${ }^{31}$ The CEM03 results agree well with the experimental fission cross sections, and better than the results of the earlier models. The Kossov approximation for the total photoabsorption cross sections in CEM03 (and CEM2k+GEM2) allows us to describe the fission cross section, not only for photon energies from $\sim 30$ 

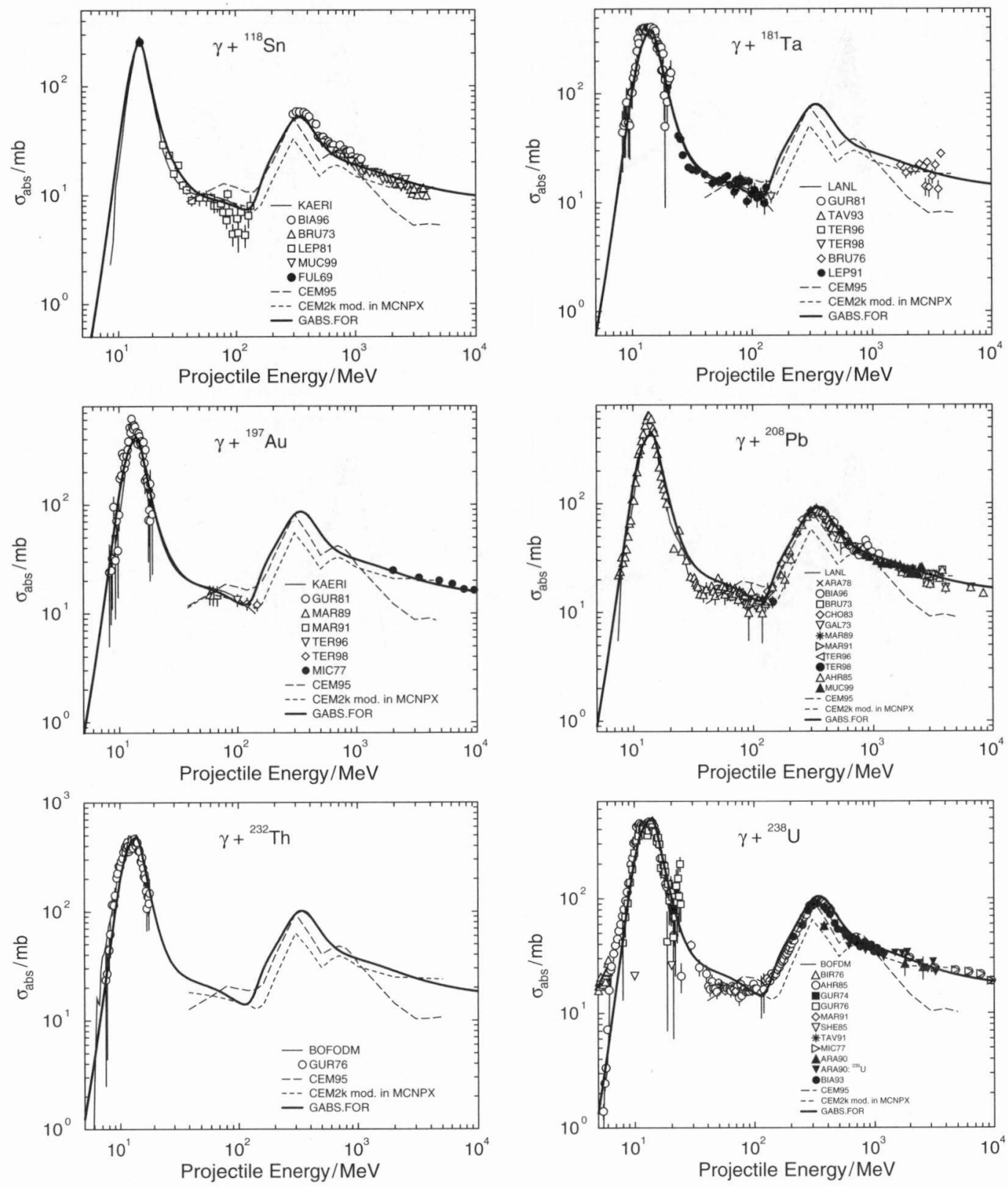

Figure 5. Examples of total photoabsorption cross sections for ${ }^{118} \mathrm{Sn},{ }^{181} \mathrm{Ta},{ }^{197} \mathrm{Au},{ }^{208} \mathrm{~Pb},{ }^{232} \mathrm{Th}$, and ${ }^{238} \mathrm{U}$ as functions of photon energy. The thick solid lines marked as "GABS.FOR" are results by our subroutine written to reproduce Kossov's ${ }^{18}$ systematics, as described in the text. The thin solid line marked as "LANL", "KAERI", or "BOFODM" show the evaluations by LANL, KAERI, or by a collaboration between IPPE/ Obninsk and CDFE/Moscow (the BOFOD(MOD) Library) from the IAEA Photonuclear Data Library. ${ }^{126}$ Results from the photonuclear version of CEM95 ${ }^{34}$ and from CEM2k as modified for MCNPX by Gallmeier ${ }^{31}$ are shown by the long-dashed and dashed lines, respectively. Experimental data (symbols) are from: BIA96, ${ }^{129}$ BRU73, ${ }^{128}$ LEP81, ${ }^{140}$ MUC99,,${ }^{133}$ FUL69, ${ }^{141}$ GUR81, ${ }^{142}$ TAV93, ${ }^{143}$ TER96, ${ }^{144}$ TER98,,${ }^{145}$ MAR89,,${ }^{146}$ MAR91, ${ }^{147}$ MIC77, ${ }^{137}$ ARA78, ${ }^{138} \mathrm{CHO} 83,{ }^{148} \mathrm{CAL} 73,{ }^{139}$ AHR85,,${ }^{127}$ GUR76, ${ }^{149}$ BIR76, ${ }^{150}$ GUR74, ${ }^{151}$ SHE85, ${ }^{152}$ TAV91, ${ }^{153}$ ARA90,,${ }^{154}$ and BIA93. ${ }^{155}$

$\mathrm{MeV}$ to $\sim 1.5 \mathrm{GeV}$, where the model is expected to be reliable, but also outside this region, i.e. in the whole range from $10 \mathrm{MeV}$ to $5 \mathrm{GeV}$.

This is an example of getting reasonably good results outside the region where the model is justified, as discussed in the previous section. Results of LAQGSM03 for these fission cross sections practically coincide with the ones by CEM03 above the GDR region, as the calculation of fission cross sections in CEM03 and LAQGSM03 were developed to be (see details in Reference 8), but are significantly lower than the data in the GDR region, as LAQGSM03 does not use the Kossov approximation and so should not be applied in the GDR region. Results of CEM95, CEM98, and CEM2k are also below the data in the GDR region, for the same reason.

We note that all the CEM03 and LAQGSM03 results shown in Figure 6 and in the following figures are obtained using default and fixed values of all parameters, without fitting anything. We only specify the energy of the incident photons and $A$ and $Z$ of the target in the inputs to CEM03 (and CEM2k+GEM2) and LAQGSM03, and then calculate. CEM95, CEM98, and CEM2k use a parameter whose value affects drastically the calculated fission cross sections, just as in many similar statistical models: This is the ratio of the level density parameters used in the fission and evaporation channels, $a_{\mathrm{f}} / a_{\mathrm{n}}$ (or, $B_{\mathrm{s}}$, in the case of CEM98, see details in Reference 158). The fission cross sections calculated by any code employing the statistical evaporation and fission models depend so much on $a_{\mathrm{f}} / a_{\mathrm{n}}$ that by fitting this ratio to available data it is possible to get a good agreement with the measured data (but not to predict unmeasured fission cross sections) with any reasonable values for the fission barriers, nuclide masses, pairing energies, and deformations, for any particular measured reaction. This is why some published papers that analyze fission cross sections or even pretend to obtain "experimental fission barriers" without addressing the question of $a_{\mathrm{f}} / a_{\mathrm{n}}$ are of low significance. In our CEM95, CEM98, and CEM2k calculations, we use the default options for nuclear masses, pairing energies, and fission barriers (the "recommended" options, in the case of CEM95, where several options are available in its input; see details in Reference 27), but we still need to define (more exactly, to fit) the values of 

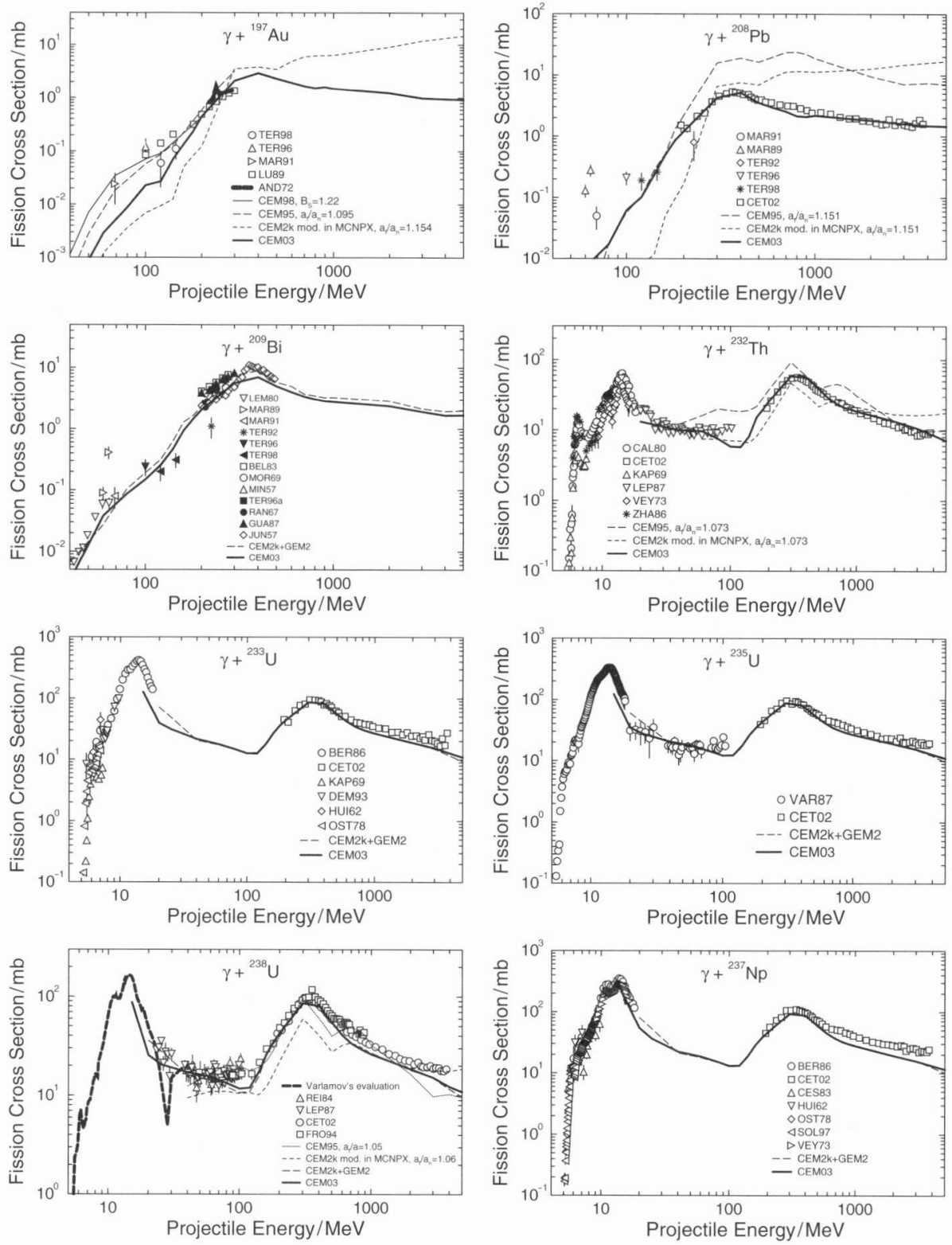

Figure 6. Comparison of the calculated photofission cross sections on ${ }^{197} \mathrm{Au},{ }^{208} \mathrm{~Pb},{ }^{209} \mathrm{Bi},{ }^{232} \mathrm{Th},{ }^{233} \mathrm{U},{ }^{235} \mathrm{U},{ }^{238} \mathrm{U}$, and ${ }^{237} \mathrm{~Np}$ with the experimental data (symbols), results by the previous versions of CEM (see details and references in the text), and the statistical evaluation by Varlamov et al. from independent measurements, ${ }^{159}$ as indicated. Experimental data are from: TER98,,${ }^{145}$ TER96, ${ }^{144}$ MAR89,${ }^{146}$ MAR91,,${ }^{147}$ LU89, ${ }^{160}$ AND72, ${ }^{161}$ TER92,,${ }^{162}$ CET02, ${ }^{163}$ LEM80 ${ }^{164}$ BEL83, ${ }^{165}$ MOR69,${ }^{166}$ MIN57, ${ }^{167}$ TER96a,${ }^{168}$ RAN67, ${ }^{169}$ GUA87, ${ }^{170}$ JUN57, ${ }^{171}$ CAL80, ${ }^{172}$ KAP69, ${ }^{173}$ LEP87, ${ }^{174}$ VEY73, ${ }^{175}$ ZHA86, ${ }^{176}$ BER86, ${ }^{177}$ DEM93, ${ }^{178}$ HUI62, ${ }^{179}$ OST78, ${ }^{180}$ VAR87, ${ }^{159}$ REI84 ${ }^{181}$ FRO $94,{ }^{182}$ CES $83,{ }^{183}$ OST78, ${ }^{180}$ and SOL $97 .{ }^{184}$

$a_{\mathrm{f}} / a_{\mathrm{n}}\left(\right.$ or, $B_{\mathrm{s}}$, in the case of CEM98): These values are listed on our plots in Figure 6. Naturally, CEM2k+GEM2, CEM03, and LAQGSM03 also had the problem of $a_{\mathrm{f}} / a_{\mathrm{n}}$ in the beginning, but this problem was solved in Reference 8 by fitting these parameters for proton-induced measured fission cross sections for all targets for which we found data, at all incident energies, and by their extrapolation/interpolation for unmeasured targets. The fitted values are fixed and are used in all our further CEM03 and LAQGSM03 calculations without subsequent variation; this allows us not only to describe well most of the measured data but also to predict reasonably well unmeasured fission cross sections. The fitting procedure ${ }^{8}$ was done so that both CEM03 (and CEM2k+GEM2) and LAQGSM03 describe as well as possible all available proton-induced measured fission cross sections; this is why the fission cross sections calculated by CEM03 practically coincide with the ones obtained by LAQGSM03 and with the experimental data.

We note that both CEM03 and LAQGSM03 assume that the reactions occur generally in three stages (see e.g. Reference 185). The first stage is the IntraNuclear Cascade (INC), in which primary particles can be re-scattered and produce secondary particles several times prior to absorption by, or escape from the nucleus. When the cascade stage of a reaction is completed, both our codes use the coalescence model described in Reference 186 to "create" high-energy d, t, ${ }^{3} \mathrm{He}$, and ${ }^{4} \mathrm{He}$ by final-state interactions among emitted cascade nucleons, already outside of the target. The emission of the cascade particles determines the particle-hole configuration, $Z, A$, and the excitation energy that is the starting point for the second, pre-equilibrium stage of the reaction. The subsequent relaxation of the nuclear excitation is treated in terms of the modified exciton model of pre-equilibrium decay followed by the equilibrium evaporation/fission stage of the reaction. Generally, all four components may contribute to experimentally measured particle spectra and other distributions. But if the residual nuclei after the INC have atomic numbers with $A \leq 12$, both CEM03 and LAQGSM03 use the Fermi break-up model described in Reference 10 to calculate their further disintegration instead of using the preequilibrium and evaporation models. The Fermi break-up is much faster than, and gives results very similar to, the continuation of the more detailed models to much lighter nuclei.

Figures 7 and 8 show two examples of proton spectra calculated by CEM03 and LAQGSM03 compared with the experimental data for the reactions $300 \mathrm{MeV} \gamma+\mathrm{Cu}^{187}$ and $198 \mathrm{MeV}$ $\gamma+\mathrm{C},{ }^{188}$ respectively. Both codes describe quite well the proton spectra in the case of copper, but less well for carbon. 


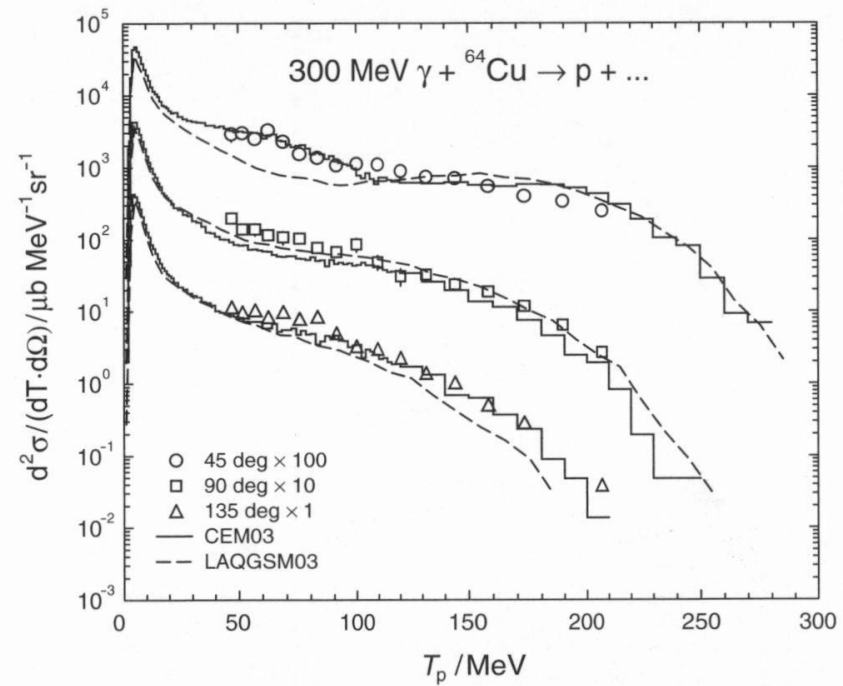

Figure 7. Proton spectra at $45^{\circ}, 90^{\circ}$, and $135^{\circ}$ from the reaction 300 $\mathrm{MeV} \gamma+\mathrm{Cu}$. Symbols are experimental data from Ref. 187, histograms and dashed lines are results of CEM03 and LAQGSM03, respectively.

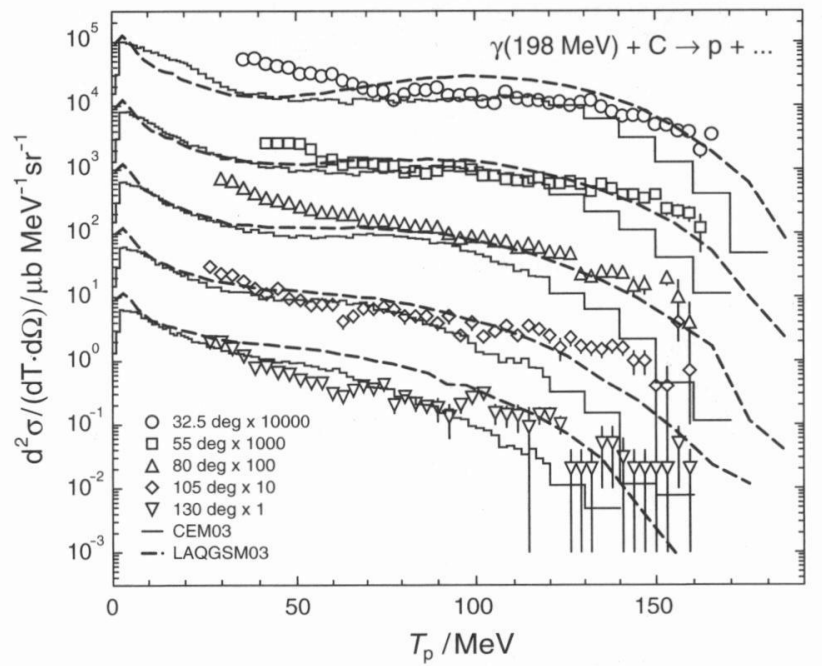

Figure 8. Proton spectra at $32.5^{\circ}, 55^{\circ}, 80^{\circ}, 105^{\circ}$, and $130^{\circ}$ from the reaction $198 \mathrm{MeV} \gamma+\mathrm{C}$. Symbols are experimental data from Ref. 188, histograms and dashed lines are results from CEM03 and LAQGSM03, respectively

Figure 9 shows examples of $\pi^{+}$angular distributions from $213 \mathrm{MeV} \gamma$ 's interacting with $\mathrm{Pb}, \mathrm{Sn}, \mathrm{Ca}$, and $\mathrm{C}$ targets. One can see that the $\pi^{+}$angular distributions calculated by CEM03 agree reasonably well with the experimental data ${ }^{189}$ for $\mathrm{C}, \mathrm{Ca}$, and $\mathrm{Sn}$ targets, but underestimate by a factor of 2 to 3 the $\mathrm{Pb}$ data. We do not have a good understanding of this disagreement. One possible explanation of this would be if CEM03 absorbs too strongly the low-energy pions produced in $\gamma \mathrm{p}$ collisions inside the target. The heavier the target the bigger would be this effect, therefore we may see it with $\mathrm{Pb}$ but not observe it for $\mathrm{C}, \mathrm{Ca}$, and $\mathrm{Sn}$ targets. There also could be problems with the experimental data for $\mathrm{Pb}$. As noted in Reference 189 , there is a systematic error in these data associated with the correction for the electron contamination in the yield for the forward detectors with $Z \geq 20$ targets. For instance, because of the magnitude of this background, no experimental cross sections are reported for the $\mathrm{Pb}$ target at $51^{\circ} .{ }^{189}$

We now consider another type of photonuclear reaction, induced by bremsstrahlung photons. In contrast to reactions induced by monoenergetic photons of a given energy $E$, the bremsstrahlung beam is produced by monoenergetic electrons and has a spectrum of photon energies $E$ of the form $N\left(E, E_{0}\right) \sim$ $1 / E,{ }^{190}$ from 0 to $E_{0}$, where the end-point energy $E_{0}$ is the

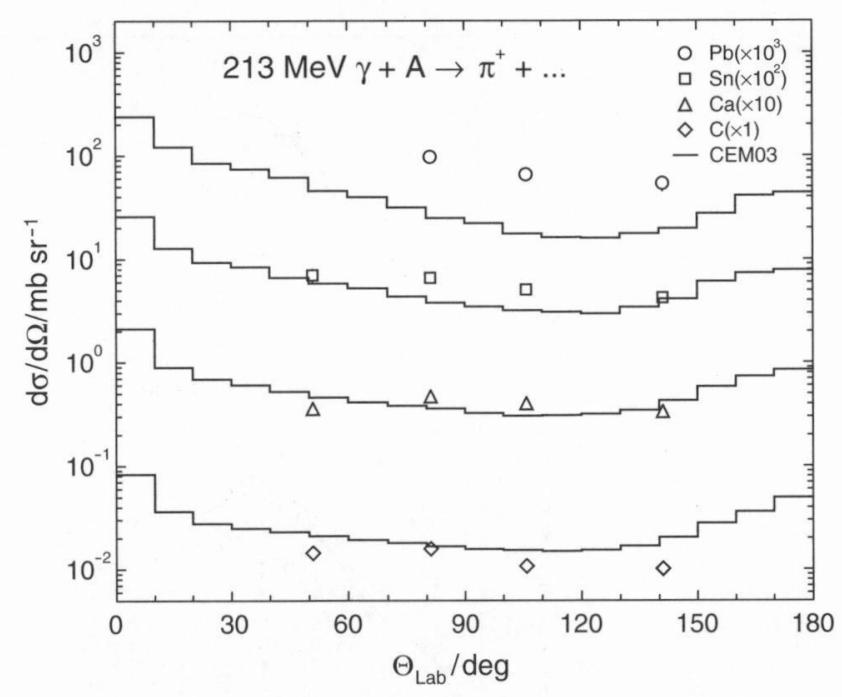

Figure 9. Energy-integrated angular distributions $\mathrm{d} \sigma / \mathrm{d} \Omega$ of $\pi^{+}$emitted from $213 \mathrm{MeV} \gamma$ interactions with $\mathrm{Pb}, \mathrm{Sn}, \mathrm{Ca}$, and C. Symbols show experimental data by Fissum et al. ${ }^{189}$ while histograms show CEM03 results.

maximum energy of photons produced by the given electron beam. In addition, all the experimental characteristics for reactions induced by bremsstrahlung photons are normalized per "equivalent quanta", $Q$, defined as:

$$
Q=\frac{1}{E_{0}} \int_{0}^{E_{0}} E \cdot N\left(E, E_{0}\right) \mathrm{d} E / \int_{0}^{E_{0}} N\left(E, E_{0}\right) \mathrm{d} E
$$

As discussed above, CEM03 and LAQGSM03 do not describe properly photonuclear reactions in the GDR region, we can calculate bremsstrahlung reactions with our codes while limiting ourselves to photon energies only above the GDR region.

This means that we need to simulate the energies of the bremsstrahlung photons according to their spectrum $N\left(E, E_{0}\right)$ $\sim 1 / E$ up to $E_{0}$ not from 0 , but from a value $E_{\min }$, above the GDR region in our calculations, and we need to use $E_{\min }$ for the lower limits of the integrals in eq 12 instead of 0 in calculating the number of equivalent quanta $Q$. This is easy to do in our Monte Carlo calculations. After simulation of $N_{\text {in }}$ numbers of interactions of bremsstrahlung gammas of energy $E_{i}$ with a nucleus, the number of equivalent quanta $Q$ will be:

$$
Q=\frac{1}{N_{\text {in }} E_{0}} \sum_{i} E_{i}=\frac{<E>}{E_{0}},
$$

where the mean energy of the bremsstrahlung photons $\langle E\rangle$ is equal to

$$
<E>=\frac{\int_{E_{\min }}^{E_{0}} E \cdot N\left(E, E_{0}\right) \mathrm{d} E}{\int_{E_{\min }}^{E_{0}} N\left(E, E_{0}\right) \mathrm{d} E}=\frac{\sum_{i} E_{i}}{N_{\text {in }}},
$$

and $E_{\min } \leq E_{i} \leq E_{0}$. In the present paper, we use $E_{\min }=30 \mathrm{MeV}$ for all the reactions we discuss. The total inelastic (photoabsorption) cross section $\sigma_{\text {in }}$ in the case of bremsstrahlung photons is calculated as following:

$$
\sigma_{\text {in }}=\frac{\int_{E_{\min }}^{E_{0}} \sigma_{\text {in }}^{\gamma}(E) \cdot N\left(E, E_{0}\right) \mathrm{d} E}{\int_{E_{\text {nin }}}^{E_{0}} N\left(E, E_{0}\right) \mathrm{d} E}=\frac{\sum_{i} \sigma_{\text {in }}^{\gamma}\left(E_{i}\right)}{N_{\text {in }}},
$$

where $\sigma_{\text {in }}^{\gamma}\left(E_{i}\right)$ is the photoabsorption cross section by a nucleus of a photon with $E_{\gamma}=E_{i}$, simulated in a particular Monte Carlo event $i$.

By using here a value of $30 \mathrm{MeV}$ instead of 0 for $E_{\min }$, we will miss the products from interaction of $\gamma$ with energies 


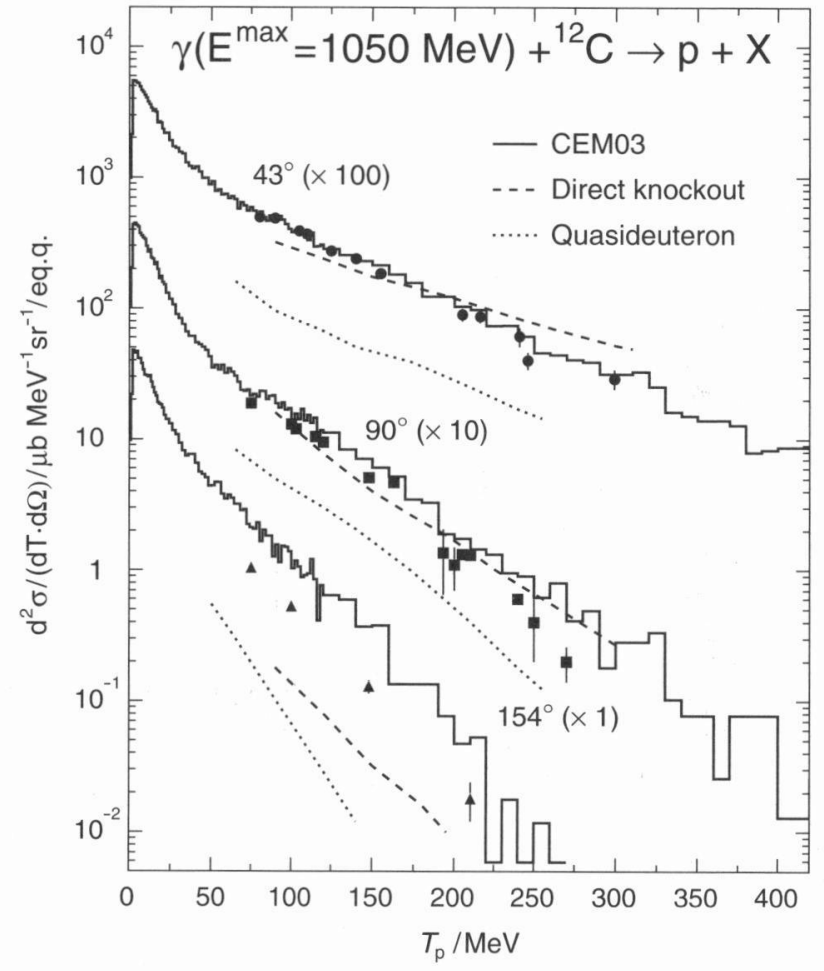

Figure 10. Comparison of measured ${ }^{191}$ differential cross section for proton photoproduction on carbon at $43^{\circ}, 90^{\circ}$, and $154^{\circ}$ by bremsstrahlung photons with $E^{\max }\left(\equiv E_{0}\right)=1.05 \mathrm{GeV}$ (symbols) with CEM03 calculations (histograms), and predictions by the direct knockout model ${ }^{192}$ (dashed lines) and a quasideuteron calculation ${ }^{193}$ (dotted lines), respectively. The experimental data and the results by the direct knockout and quasi-deuteron models were taken from Figure 5 of Ref. 192.

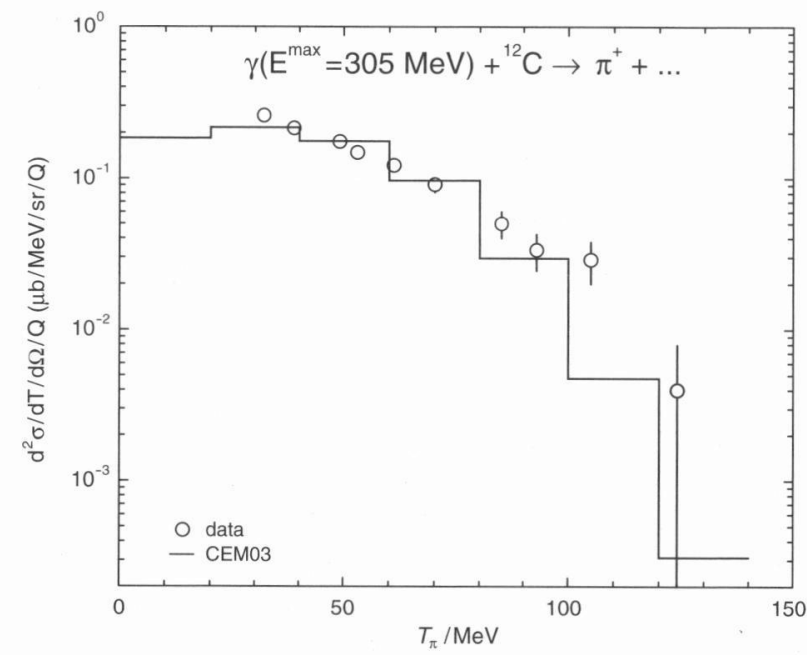

Figure 11. Comparison of measured ${ }^{194}$ differential cross section for $\pi^{+}$ photoproduction on carbon at $90^{\circ}$ by bremsstrahlung photons with $E^{\max }\left(\equiv E_{0}\right)=305 \mathrm{MeV}$ (circles) with CEM03 calculations (histogram).

below $30 \mathrm{MeV}$, like the cross sections for $(\gamma, \mathrm{n}),(\gamma, 2 \mathrm{n})$, and, to a certain degree, $(\gamma, \mathrm{np})$ in our results, but this limitation does not affect at all the description of products in the deep spallation, fission (for preactinides), and fragmentation regions, as well as the pion photoproduction and spectra of nucleons and complex particles at energies above the evaporation region.

Figures 10 and 11 present examples of proton and $\pi^{+}$spectra from bremsstrahlung interaction with carbon at $E_{0}=1050$ and $305 \mathrm{MeV}$, respectively. One can see that CEM03 describes well both the measured proton and pion spectra and agrees with the data better than the direct knockout model ${ }^{192}$ and the quasi-deuteron calculations ${ }^{193}$ do.

Since the 1980s, a large number of radiochemical measurements of bremsstrahlung-induced reactions have been performed in Japan by the group of Sakamoto (see the recent reviews ${ }^{195}$, 196
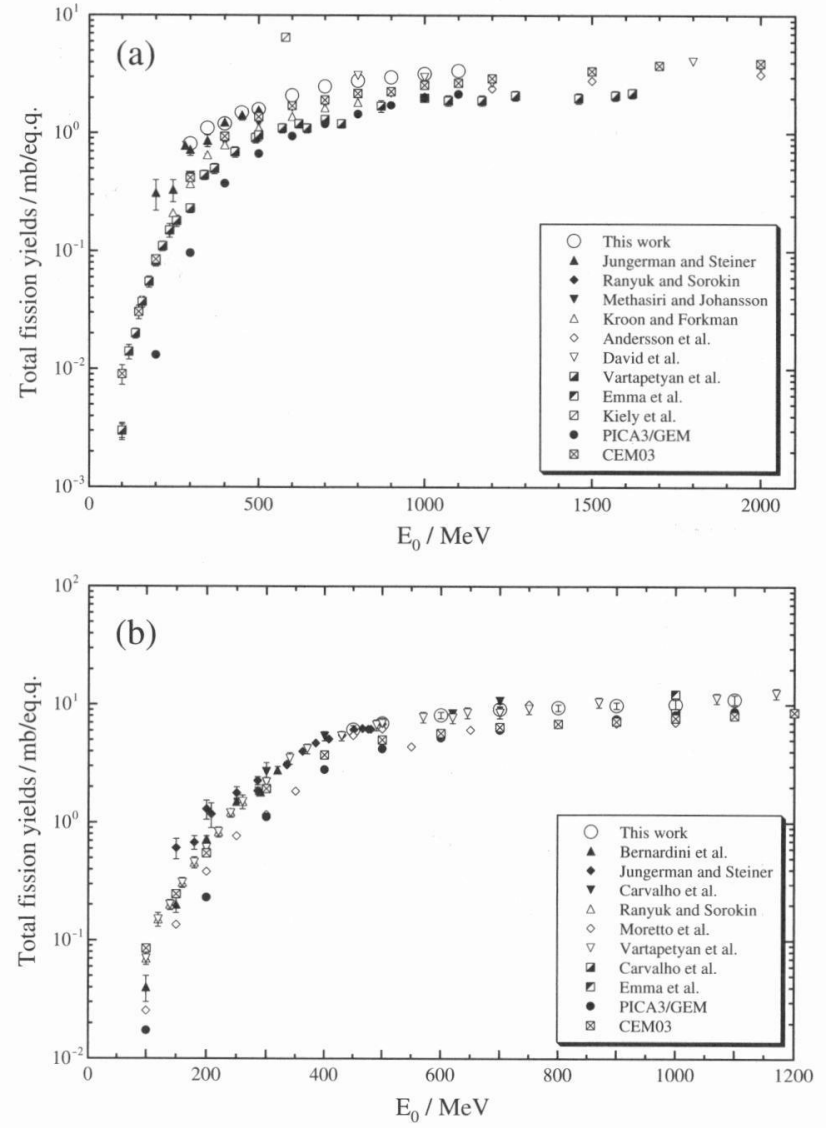

Figure 12. Bremsstrahlung-induced fission cross sections of ${ }^{197} \mathrm{Au}$ (a) and ${ }^{209} \mathrm{Bi}(\mathrm{b})$ as functions of the end-point energy $E_{0}$. The experimental data for Au indicated in the insert of the figure as "This work" are from Ref. 202; other experimental data on Au are from Refs. 169, 171, and 204-209, as indicated. The data for $\mathrm{Bi}$ indicated as "This work" are from Ref. 203 and other data for Bi are from Refs. 166, $169,171,207,208$, and $210-212$, as indicated. The PICA3/GEM ${ }^{201}$ results shown with filled circles are from Ref. 203; our present CEM03 results are shown with crossed squares. We thank Haba for making this figure for us by adding our CEM03 results to Figure 19 of the review. ${ }^{195}$

and references therein). Thousands of useful product cross sections were measured by this group on target nuclei from ${ }^{7} \mathrm{Li}$ to ${ }^{209} \mathrm{Bi}$ at bremsstrahlung end-point energies $E_{0}$ from $30 \mathrm{MeV}$ to $1.2 \mathrm{GeV}$, including photopion reactions, fragmentation and fission of preactinides, deep spallation reactions, and recoil studies (mean kinetic energy and the forward/backward (F/B) ratios of products). The authors of these measurements have analyzed most of their data with the PICA code by Gabriel et al. ${ }^{197,198}$ with its improved version PICA95, ${ }^{199,200}$ as well as with its latest version, PICA3, merged ${ }^{201}$ with the GEM evaporationfission code by Furihata ${ }^{9}$ mentioned above, i.e. PICA3/GEM.

Recently, this group provided us with numerical values of some of their measured reactions and we have calculated them with CEM03. Figure 12 shows an example of the comparison of CEM03 results with data for bremsstrahlung-induced fission cross sections of ${ }^{197} \mathrm{Au}^{202}$ and ${ }^{209} \mathrm{Bi}^{203}$ compared as well with other available experimental data for $\mathrm{Au}^{169,171,204-209}$ and for $\mathrm{Bi},{ }^{166,169,171,207,208,210-212}$ and with results by PICA3/GEM ${ }^{201}$ from Reference 203. There is a reasonable agreement of the CEM03 results with the experimental data in the whole interval of $E_{0}$ measured, from the threshold to the highest measured energy.

Figure 13 presents the experimental data ${ }^{202,203,213-216}$ and the calculations by PICA3/GEM ${ }^{201}$ and by CEM03 for the mass yields of products produced by bremsstrahlung reactions on ${ }^{197} \mathrm{Au}$ and ${ }^{209} \mathrm{Bi}$ at $E_{0}=1 \mathrm{GeV}$. For convenience, all the isotopes produced in these reactions were divided into four groups, namely: 1) spallation products produced by sequential emission of several nucleons, positive pions, and complex 

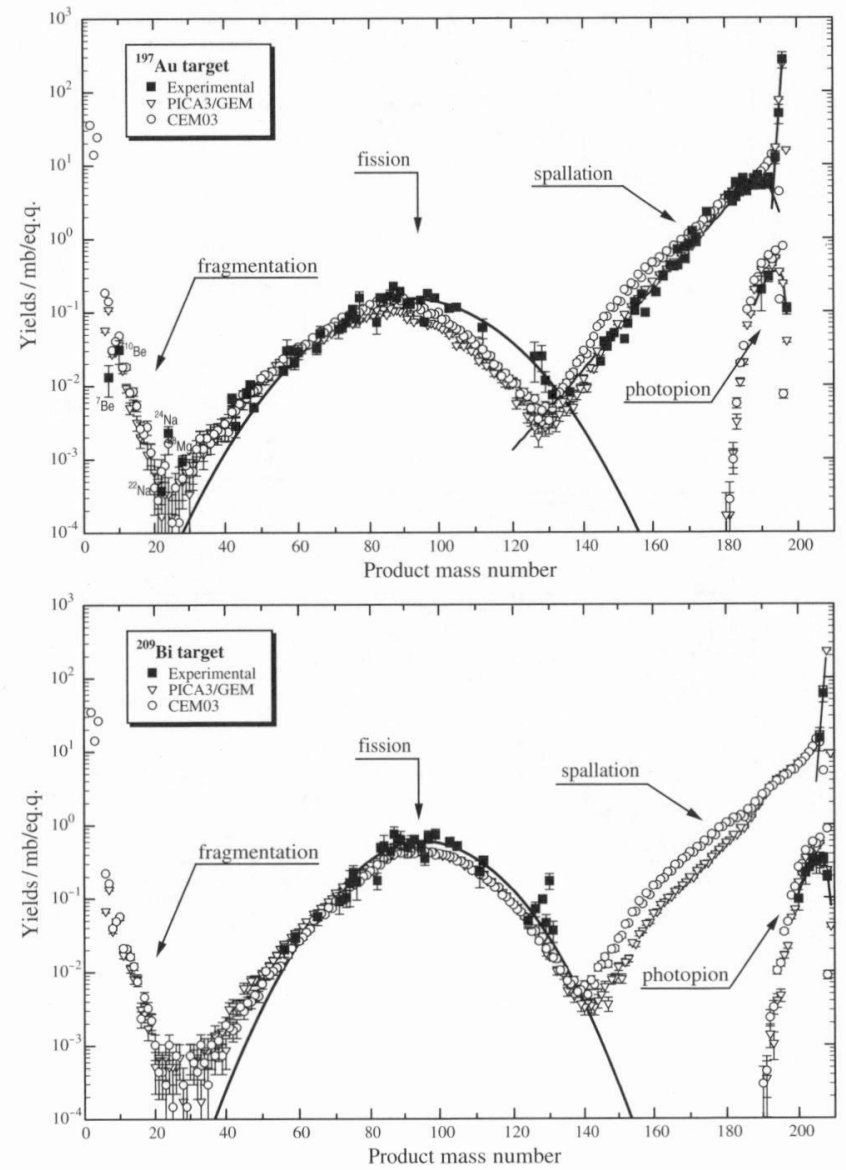

Figure 13. Comparison of CEM03 results for the mass yields of products produced by bremsstrahlung reactions on ${ }^{197} \mathrm{Au}$ and ${ }^{209} \mathrm{Bi}$ at $E_{0}=1$ $\mathrm{GeV}$ with the experimental data ${ }^{202,203,213-216}$ and the calculations by PICA3/GEM. ${ }^{201}$ The experimental yields from fission of ${ }^{197} \mathrm{Au}$ and ${ }^{209} \mathrm{Bi}$ are from Ref. 213; those by spallation on ${ }^{197} \mathrm{Au}$ are from Refs. 214 and 216; those by fragmentation of ${ }^{197} \mathrm{Au}$ are from Ref. 215; the PICA3/GEM results are from several publications of Sakamoto's group and are presented in Figure 18 of the review ${ }^{195}$ with the corresponding citations. The mass yields for the fission products shown by black curves represent approximations based on the experimental data obtained in Refs. 202 and 203. We thank Matsumura for making this figure for us by adding our CEM03 results to Figure 18 of the review. ${ }^{195}$

particles during the INC, followed by preequilibrium and evaporation processes; 2 ) intermediate-mass nuclides produced via fission of excited compound nuclei; 3) light fragments emitted either via evaporation or by "fragmentation" (Fermi break-up model, in the case of our present results), and 4) "photopion" products produced in $\left(\gamma, \pi^{-} x \mathrm{n}\right)$ and $\left(\gamma, 2 \pi^{-} x \mathrm{n}\right)$ reactions, where the charge of the products is higher than that of the initial target. One can see that CEM03 describes quite well the yields of products in all these groups and agrees with the experimental data and results by PICA3/GEM. CEM03 does not describe well the spallation products very near the target, that are produced via $(\gamma, x \mathrm{n})$ reactions, because it does not consider photons with energies in the GDR region $\left(E_{\min }=30 \mathrm{MeV}\right)$, as discussed above.

Some of the experimental data points in the spallation region (in the case of ${ }^{197} \mathrm{Au}$ ) are overestimated by calculations up to a factor of two or three, while some of the experimental yields in the fission region are underestimated up to a factor of five or more. We do not have yet a clear understanding of this disagreement and we plan to address it in the future. Comparing our calculation results with experimental data isotope-by-isotope, as we did earlier for proton-induced reactions (see, e.g. Reference 3 and 217 and references therein), rather than the mass distributions, may help us understand better and solve this problem. We plan to perform such an analysis of isotopic yields from photonuclear reactions in the future.

Our preliminary analysis shows that CEM03 also allows us
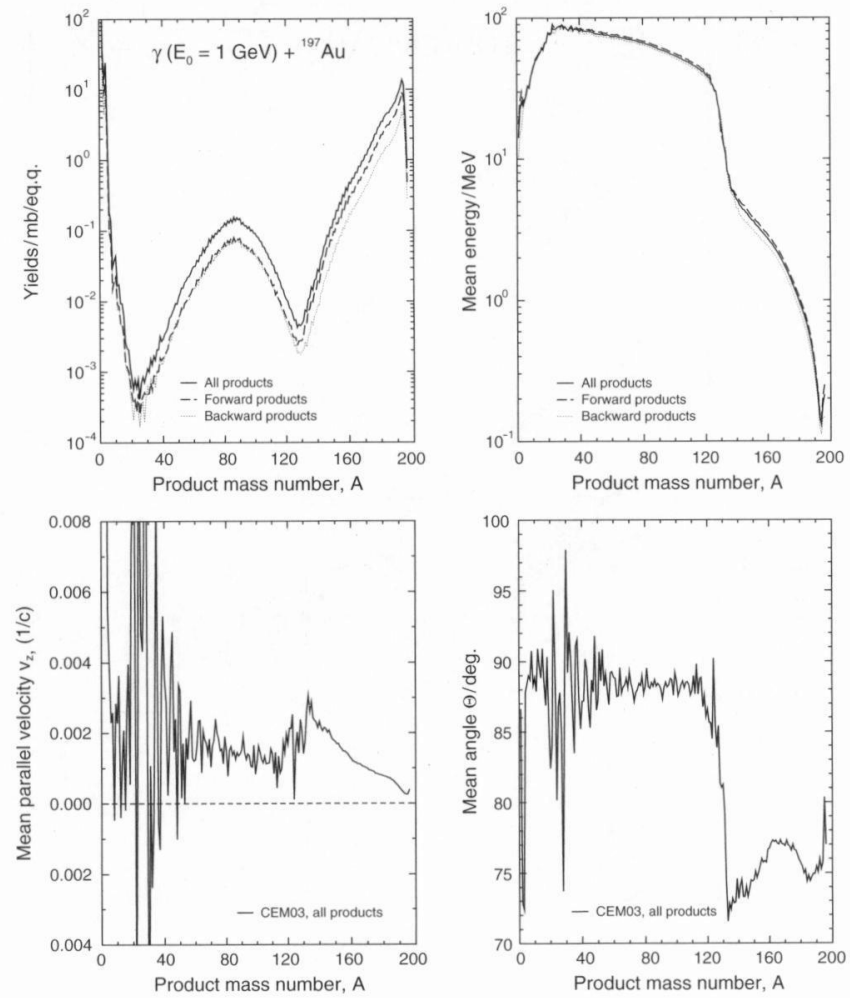

Figure 14. Results by CEM03 for $1 \mathrm{GeV}$ bremsstrahlung-induced reactions on $\mathrm{Au}$. Upper left plot: mass yield of all products (thick solid line), isotopes produced in the forward laboratory direction (thick long-dashed line), and backward products (thin dotted line); Upper right plot: mean laboratory kinetic energy of all products (solid line) and of only forward (long-dashed line) and backward products (dotted line); Lower left plot: mean laboratory velocity $v_{z}$ of all products in the beam direction; Lower right plot: mean laboratory angle $\Theta$ of all products as a function of $A$. The big fluctuations in the values of $v_{z}$ and $\Theta$ for masses around $A=20$ and 130 do not provide real physical information, as they are related to the limited statistics of our Monte Carlo simulation caused by the very low yield of isotopes at the border between spallation and fission, and at that between fission and fragmentation. Our calculation provides only a few (or even one) isotopes of a given $A$ in these mass regions, and mean values for such events do not have any significance.

to describe the recoil properties (forward and backward product yields, their $\mathrm{F} / \mathrm{B}$ ratio, and mean kinetic energies) of nuclides produced in bremsstrahlung-induced reactions on medium and heavy targets at intermediate energies (see Reference 196 and reference therein). We plan to publish our analysis in a future paper. Below, we present only several predictions by CEM03 for the bremsstrahlung-induced reaction on $\mathrm{Au}$ at $E_{0}=1 \mathrm{GeV}$, as we find such results informative and useful to better understand the mechanisms of nuclear reactions.

Due to the momentum transferred by the bombarding gammas to the nuclear target, one may expect that most of the spallation products would fly in the forward direction in the laboratory system. The lower-right plot in Figure 14 shows the mean laboratory angle $\Theta$ of all products as a function of $A$. We see that the mean angle of the spallation products is predicted by CEM03 to be between 72 and 80 degrees. (It is not equal to 0 degrees, as the probability of projectiles to have an impact parameter exactly equal to zero is equal to zero, and photons hit more often the periphery of the nucleus rather than its center.) The thick solid curve in the upper-left plot of Figure 14 shows the yield of all products as a function of $A$, the same results compared in Figure 13 with the experimental data and the calculations by PICA3/GEM. Besides the total yield, this plot shows also its components from nuclides produced in the forward (thick long-dashed line) and backward (thin dotted line) directions. One can see that for all the spallation isotopes, the cross sections for the forward products are about a factor of two higher than those for backward products, 


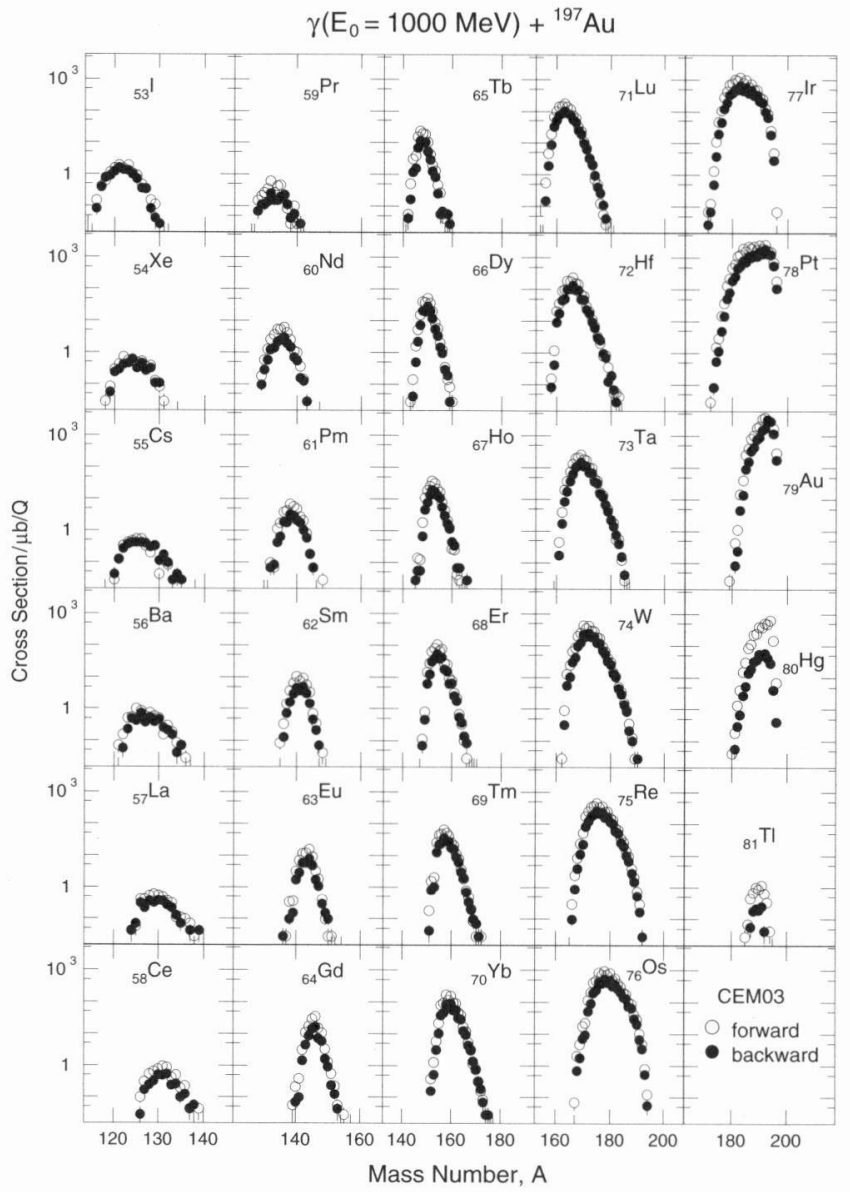

Figure 15. Predicted cross sections of the spallation products from the $E_{0}=1 \mathrm{GeV}$ bremsstrahlung photon-induced reaction on Au: Open circles show the yield of the products produced in the forward direction in the laboratory system, while the black circles show results for the backward products.

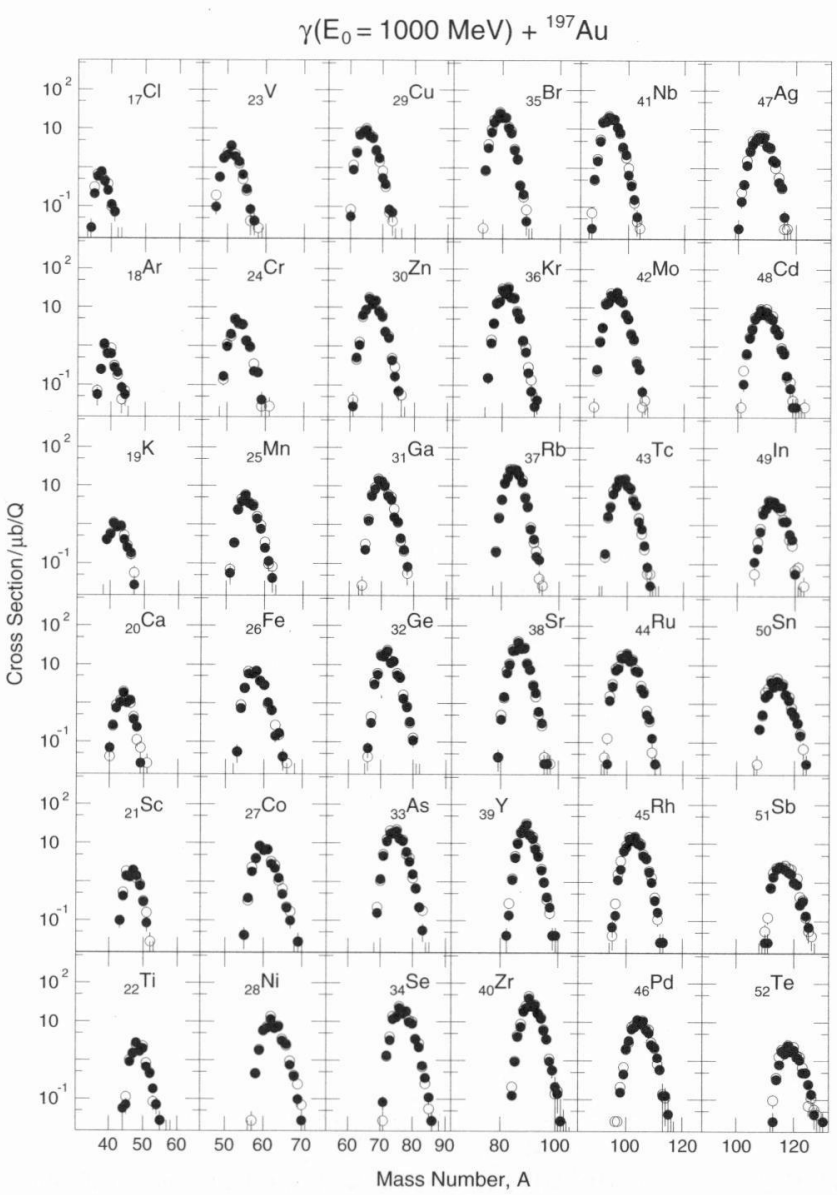

Figure 16. The same as Figure 15, but for fission and fragmentation products.
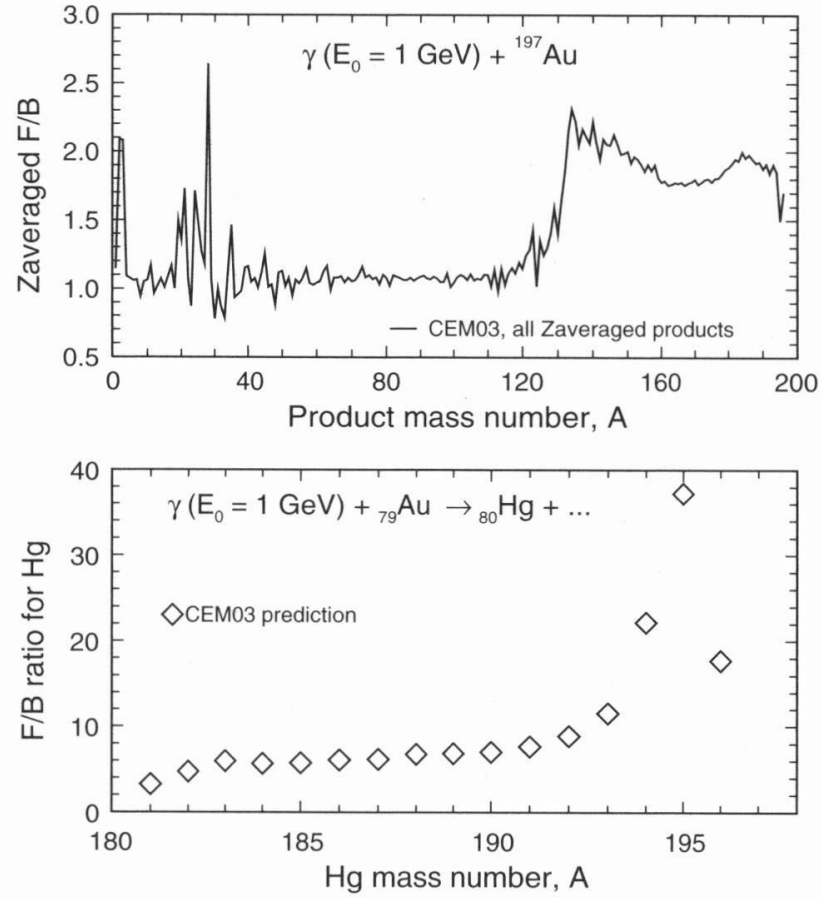

Figure 17. Upper plot: $Z$-averaged $A$-dependence of the $\mathrm{F} / \mathrm{B}$ ratio of the forward product cross sections to the backward ones, as predicted by CEM03 for the reaction of $E_{0}=1 \mathrm{GeV}$ bremsstrahlung photons on Au. Lower plot: The F/B ratio of the $\mathrm{Hg}$ isotopes produced in the same reaction as a function of the mass number of the $\mathrm{Hg}$ nuclei.

in complete accordance with the available experimental data (see the review ${ }^{196}$ and references therein). But the situation changes completely for fission products: The momenta of the fissioning nuclei is small, their mean kinetic energy in the laboratory system is a few $\mathrm{MeV}$ (see the upper-right plot in Figure 14), that is much less than the kinetic energy from several tens to about a hundred $\mathrm{MeV}$ that fission fragments receive due to the Coulomb repulsion of the fragments. If we neglect the effects of angular momentum, the fission fragments would be distributed isotropically in the system of the fissioning nucleus, and the small momentum of the fissioning nuclei makes this distribution almost isotropic also in the laboratory system. The upper left plot of Figure 14 shows that the predicted yields for the fission fragments in the forward and backward directions are almost the same, i.e. the F/B ratio for the fission fragments is almost equal to one, again in complete agreement with the available experimental data (see Reference 196 and references therein).

The mean kinetic energy of the forward products shown in the upper-right plot of Figure 14 is only very slightly higher than that of the backward products (the momenta of fissioning nuclei are low, as discussed above), with a little higher effect for the spallation products than for fission fragments, as is to be expected. Due to this fact and to the near isotropy of the fission fragments, some fission fragments may have their mean velocity in the direction opposite the beam, as can be seen from in the lower-left plot of this figure.

It is much more informative to study the F/B problem considering the forward and backward cross sections for every product separately, as shown in Figures 15 and 16, rather than addressing only the $A$-distribution of their yields. Whereas the $Z$-averaged $A$-dependence of the $\mathrm{F} / \mathrm{B}$ ratio is about a factor of two for all the spallation region (see also Figure 17), the situation changes for individual isotopes. The cross sections of the forward-emitted isotopes are still about a factor of two higher than the backward cross sections for most of the spallation products, but their ratio is much higher for $\mathrm{Hg}$ and $\mathrm{Tl}$, and depends strongly on the mass numbers of the products. $\mathrm{Hg}$ and $\mathrm{Tl}$ are "photopion" products produced via $\left(\gamma, \pi^{-} x \mathrm{n}\right)$ and $(\gamma$, $\left.2 \pi^{-} x \mathrm{n}\right)$, respectively, with emission of only a few neutrons in 
addition to the pions. When the number of emitted neutrons is small, the product "remembers" the momentum transfered to the target by the projectile, and such neutron-rich products go mainly forward, with a ratio $F / B$ up to ten or higher. On the contrary, in reactions where many neutrons are emitted approximately isotropically, the residual nucleus has lost most of its "memory" of the initial momentum. Therefore the neutron-deficient products from such reactions have a smaller F/B ratio, usually around a factor of two. The farther away from the target are the products, the smaller is this effect; for products with $Z \leq 70$, it practically disappears. Approaching the border around $A=130$ of the transition between spallation and fission products, the $\mathrm{F} / \mathrm{B}$ ratio decreases and for $\mathrm{Ce}, \mathrm{La}$, $\mathrm{Ba}, \mathrm{Cs}, \mathrm{Xe}$, and I nuclei shown in the left column of Figure 15, the F/B ratio becomes almost equal to one and remains so for all the fission products shown in Figure 16.

The $Z$-averaged F/B ratio for all nuclides of a given $A$ as a function of $A$ is presented in the upper plot of Figure 17. The lower plot of this figure show the F/B ratio for isotopes of $\mathrm{Hg}$ : One can see that it decreases from about thirty-seven for neutron-rich ${ }^{195} \mathrm{Hg}$ produced by the ${ }^{197} \mathrm{Au}\left(\gamma, \pi^{-2} 2 \mathrm{n}\right)$ reaction to about three for neutron-deficient ${ }^{181} \mathrm{Hg}$ produced by the ${ }^{197} \mathrm{Au}\left(\gamma, \pi^{-} 16 \mathrm{n}\right)$ reaction.

We think that an analysis of such recoil characteristics is quite informative not only for photonuclear reactions, but also for proton-induced and other types of reactions. Analyses of the experimental data for such characteristics would allow us to understand better the mechanisms of nuclear reactions and may help us to distinguish the fission processes from the fragmentation (or evaporation) ones in the production of heavy fragments from reactions on medium-mass targets, like $\mathrm{Fe}$ (see discussion of this problem in References 1 and 2). New measurements on the recoil properties from reactions with any type of projectiles, including bremsstrahlung photons, would be very useful.

\section{Conclusions}

The 2003 versions of the codes CEM $2 k+$ GEM2 and LAQGSM, CEM03 and LAQGSM03, are extended to describe photonuclear reactions. Both our models consider photoproduction of at most two pions, which limits their reliable application to photon energies up to only about $1.5 \mathrm{GeV}$. The present version of our models do not consider photoabsorption in the GDR region, which defines the lower limit of the photon energy to about $30 \mathrm{MeV}$. Nevertheless, developing and incorporating of a routine based on the phenomenological systematics for the total photoabsorption cross section by Kossov into CEM03 allow us to enlarge the region of applicability of CEM03 and to get quite reasonable results for applications both in the GDR region and above $1.5 \mathrm{GeV}$.

As shown by several examples, CEM03 and LAQGSM03 allow us to describe reasonably well, and better than their precursors, many photonuclear reactions needed for applications, as well as to analyze mechanisms of photonuclear reactions for fundamental studies. But our models still have several problems. Figure 18 shows examples of such problems on proton and deuteron spectra from reactions induced by 60 $\mathrm{MeV}$ monoenergetic photons on $\mathrm{Ca}$ : One can see that both CEM03 and LAQGSM03 describe reasonably well the shape of the proton spectrum, but their absolute values differ by more than a factor of two. This is because the CEM03 results are normalized to the total photoabsorption cross section predicted

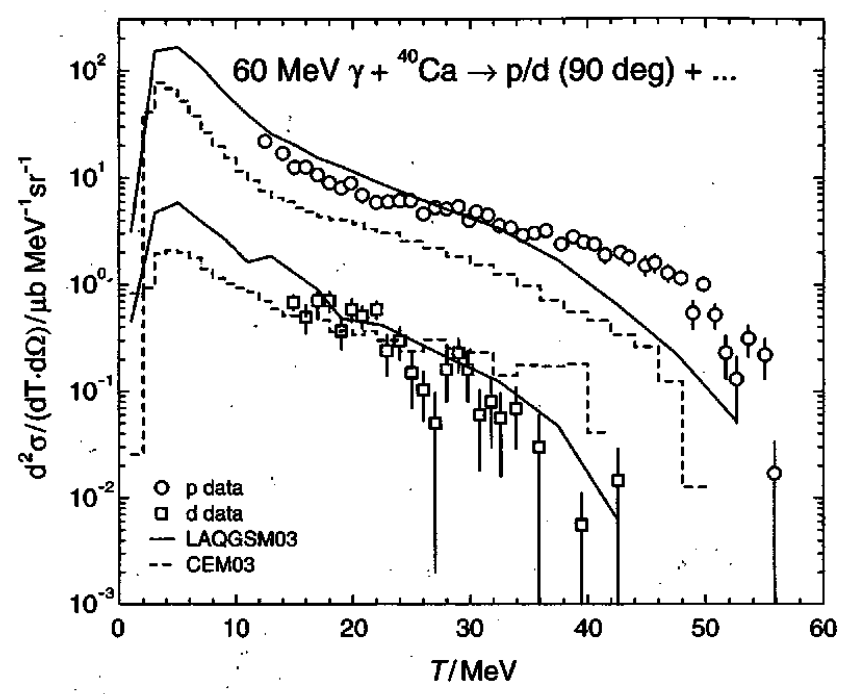

Figure 18. Examples of two problems with the current versions of our codes: proton and deuteron spectra at $90^{\circ}$ from the reaction $60 \mathrm{MeV} \gamma$ + Ca. Symbols are experimental data from Ref. 218, solid lines and dashed histograms are results from LAQGSM03 and CEM03, respectively. The CEM03 spectra are normalized to the total absorption cross section as predicted by Kossov's systematics, equal to $3.49 \mathrm{mb}$ for this reaction, while the LAQGSM03 spectra are normalized to the Monte-Carlo total absorption cross section calculated by that code to be equal to $8.5 \mathrm{mb}$.A

by the Kossov systematics, which gives $3.49 \mathrm{mb}$ for this reaction, while the LAQGSM03 results are normalized to the MonteCarlo-calculated total photoabsorption cross section of $8.5 \mathrm{mb}$. If we refer to Figure 4, we see that the Kossov systematics for the reaction $\gamma+$ Ca predicts. values that are a factor of two below the available experimental data at energies around 60 $\mathrm{MeV}$. This explains the difference between the CEM03 and LAQGSM03 results shown in Figure 18, and suggest that the Kossov systematics should be further improved. Even allowing for this normalization problem, both codes appear to underestimate the cross sections for the higher-energy protons.

A further problem shown in Figure 18 is for the spectra of deuterons. The'predicted spectra of deuterons differ both in their shapes and absolute values for the two codes. CEM03 and LAQGSM03 have different intranuclear-cascade models, leading after the INC stage of any reactions to different average values for $A, Z$, and $E$ of the excited nuclei, as a starting point for the preequilibrium and evaporation stages of reactions, where most of the deuterons are produced. This explains the difference in the deuteron spectra predicted by the two codes and suggests that further work to improve the description of complex-particle emission is necessary.

The overestimation of the high-energy tail of the deuteron spectrum by CEM03 is partially related with an imperfect description of the preequilibrium emission of $d$ from this reaction, due to an excessively simplified estimation of the probability of several excited nucleons (excitons) coalescing into a complex particle that can be emitted during the decay of the excited nuclei produced after the cascade.

We plan to address these problems in the future. In addition, we plan to extend our models to describe photoabsorption in the GDR region, as discussed previously, and to extend our models to describe photonuclear reactions at energies of 10 $\mathrm{GeV}$ or more. ${ }^{\dagger}$

Our present study suggests that analysis of characteristics of

\footnotetext{
${ }^{\dagger}$ Actually, after completing the manuscript of the present paper, we have extended already LAQGSM03 to describe photonuclear reactions on nuclei to higher energies, up to $10 \mathrm{GeV}$ and higher. This version of LAQGSM is called LAQGSM03.01. It considers 56 different channels for elementary $\gamma_{\mathrm{p}}$ interactions and 56 channels for similar $\gamma_{\mathrm{n}}$ interactions and it is described briefly in our recent LANL paper: S. G. Mashnik, K. K. Gudima, M. I. Baznat, A. J. Sierk, R. E. Prael, and N. V. Mokhov, "CEM03.01 and LAQGSM03.01 Versions of the Improved Cascade-Exciton Model (CEM) and Los Alamos Quark-Gluon String Model (LAQGSM) Codes," Los Alamos National Laboratory Research Note X-5-RN (U) 0511, LANL Report LA-UR-05-2686 (2005). LAQGSM03.01 will be described in details in a separate future paper.
} 
recoil nuclei produced by photonuclear and other types of reactions is a powerful tool to understand mechanisms of nuclear reactions. We encourage future measurements of such characteristics both for photonuclear and proton-or/and nucleusinduced reactions.

Acknowledgments. We thank Dr. Kumataro Ukai for providing us with numerical values of single pion photoproduction cross sections from their compilation. ${ }^{36}$ We are grateful to Dr. Igor Pshenichnov for sending us the $\gamma \mathrm{p}$ and $\gamma \mathrm{n}$ event generators used in their Moscow photonuclear reaction INC. ${ }^{33}$ We thank Prof. Koh Sakamoto and Drs. Hiroshi Matsumura, Hiromitsu Haba, and Yasuji Oura for providing us with their publications and numerical tables of their measured data, as well as for useful discussions, help in drawing several figures for us, and their interest in our modeling.

This work was partially supported by the Advanced Simulation Computing (ASC) Program at the Los Alamos National Laboratory operated by the University of California for the US Department of Energy, the Moldovan-US Bilateral Grants Program, CRDF Project MP2-3045-CH-02, and the NASA ATP01 Grant NRA01-01-ATP-066.

\section{References}

(1) S. G. Mashnik, K. K. Gudima, A. J. Sierk, and R. E. Prael, Los Alamos National Laboratory Report LA-UR04-0039 (2004); Proc. ND2004, Santa Fe, USA, 2004, ed. R. C. Haight, M. B. Chadwick, T. Kawano, and P. Talou, (AIP Conf. Proc. 769, Melville, New York, 2005), p. 1188; E-print: nucl-th/0502019.

(2) S. G. Mashnik, K. K. Gudima, R. E. Prael, and A. J. Sierk, Los Alamos National Laboratory Report LA-UR03-7277 (2003).

(3) S. G. Mashnik, K. K. Gudima, R. E. Prael, and A. J. Sierk, Proc. Workshop on Nuclear Data for the Transmutation of Nuclear Waste, GSI-Darmstadt, Germany, 2003, ISBN 300-012276-1, ed. A. Kelic and K.-H.Schmidt, http://wwwwnt. gsi.de/tramu/; E-print: nucl-th/0404018.

(4) S. G. Mashnik and A. J. Sierk, Proc. AccApp2000, Washington DC, USA, 2000, (ANS, La Grange Park, USA, 2001), p. 328; E-print: nucl-th/0011064.

(5) K. K. Gudima, S. G. Mashnik, and V. D. Toneev, Nucl. Phys. A 401, 329 (1983).

(6) S. G. Mashnik, K. K. Gudima, and A. J. Sierk, Proc. SATIF6, SLAC, USA, 2002, (NEA/OECD, Paris, France, 2004), p. 337; E-print: nucl-th/0304012.

(7) S. G. Mashnik, A. J. Sierk, and K. K. Gudima, Los Alamos National Laboratory Report LA-UR-02-5185 (2002); Eprint: nucl-th/0208048.

(8) M. Baznat, K. Gudima, and S. Mashnik, Los Alamos National Laboratory Report LA-UR-03-3750 (2003); Proc. AccApp'03, San Diego, USA, 2003, (ANS, La Grange Park, USA, 2004), p. 976; E-print: nucl-th/0307.014.

(9) S. Furihata, Nucl. Instr. Meth. B 171, 252 (2000); Ph.D. thesis, Tohoku University (2003).

(10) K. K. Gudima, S. G. Mashnik, and A. J. Sierk, Los Alamos National Laboratory Report LA-UR-01-6804 (2001).

(11) N. V. Mokhov, K. K. Gudima, C. C. James, M. A. Kostin, S. G. Mashnik, E. Ng, J.-F. Ostiguy, I. L. Rakhno, A. J. Sierk, and S. I. Striganov, Los Alamos National Laboratory Report LA-UR-04-3047 (2004); E-print: nucl-th/0404084; Proc. ICRS-10/RPS2004 Int. Conf., Funchal, Madeira, Portugal, 2004, in press.

(12) R. E. Prael, Los Alamos National Laboratory Report LAUR-01-1655 (2001).

(13) J. S. Hendricks, G. W. McKinney, L. S. Waters, T. L. Roberts, H. W. Egdorf, J. P. Finch, H. R. Trellue, E. J. Pitcher, D. R. Mayo, M. T. Swinhoe, S. J. Tobin, J. W.
Durkee, F. X. Gallmeier, J.-C. David, W. B. Hamilton, and J. Lebenhaft, Los Alamos National Laboratory Report LA-UR-04-0570 (2004) and references therein; http://mcnpx.lanl.gov/.

(14) F. B. Brown, Los Alamos National Laboratory Report LAUR-04-2648 (2004), Proc. PHYSOR-2004, 4-29-2004; R. A. Forster, L. J. Cox, R. F. Barrett, T. E. Booth, J. F. Breismeister, F. B. Brown, J. S. Bull, G. C. Geisler, J. T. Goorley, R. D. Mesteller, S. E. Post, R. E. Prael, E. C. Selcow, and A. Sood, Nucl. Instrum. Methods B 213, 82 (2004); J. F. Briesmeister, Ed., Los Alamos National Laboratory Report LA-13709-M (2000); www-xdiv.lanl. gov/x $5 / \mathrm{MCNP} /$.

(15) K. K. Gudima, A. S. Iljinov, and V. D. Toneev, Communication JINR P2-4661, Dubna (1969).

(16) V. S. Barashenkov and V. D. Toneev, Interaction of High Energy Particles and Nuclei with Atomic Nuclei, Atomizdat, Moscow (1972).

(17) V. S. Barashenkov, F. G. Geregi, A. S. Iljinov, G. G. Jonsson, and V. D. Toneev, Nucl. Phys. A 231, 462 (1974).

(18) M. V. Kossov, Eur. Phys. J. A 14, 377 (2002).

(19) J. S. Levinger, Phys. Rev. 84, 43 (1951); Nuclear PhotoDisintegration (Oxford University Press, 1960); Phys. Lett. B 82, 181 (1979).

(20) W. O. Lock and D. F. Measday, Intermediate Energy Nuclear Physics (London, Methuen, Distributed in the U.S.A. by Barnes and Noble, 1970).

(21) V. S. Barashenkov, K. K. Gudima, and V. D. Toneev, JINR Communication P2-4065, Dubna (1968).

(22) V. S. Barashenkov, K. K. Gudima, and V. D. Toneev, JINR Communication P2-4066, Dubna (1968); Acta Physica Polinica 36, 415 (1969).

(23) S. Eidelman et al. (Particle Data Group), Phys. Lett. B 592, 1 (2004); http://pdg.lbl.gov.

(24) S. J. Lindenbaum and R. M. Sternheimer, Phys. Rev. 105, 1874 (1957).

(25) V. S. Barashenkov, Le Van Ngok, L. G. Levchuk, Zh. Zh. Musul'manbekov, A. N. Sosnin, V. D. Toneev, and S. Yu. Shmakov, JINR Communication R2-85-173, Dubna (1985); V. S. Barashenkov, Comp. Phys. Commun. 126, 28 (2000).

(26) N. Amelin, Preprint CERN/IT/99/6, CERN, Geneva (1999); http://atlas.web.cern.ch/Atlas/ GROUPS/SOFTWARE/OO/domains/simulation/G4PhysicsStudies/documentation/ameline/ makebookphysics.html\#tex $2 \mathrm{html} 1$.

(27) S. G. Mashnik, User Manual for the Code CEM95 (JINR, Dubna, USSR, 1995); OECD NEA Data Bank, Paris, France; http://www.nea.fr/abs/html/iaea1247.html; RSICPSR-357 (Oak Ridge, USA, 1995).

(28) S. G. Mashnik and A.J. Sierk, Proc. SARE-4, Knoxville, TN, 1998, ed. T. A. Gabriel, (ORNL, 1999), p. 29; Eprint: nucl-th/9812069.

(29) N. V. Mokhov, S. I. Striganov, A. Van Ginneken, S. G. Mashnik, A. J. Sierk, and J. Ranft, Proc. SARE-4, Knoxville, TN, 1998, ed. T. A. Gabriel, (ORNL, 1999), p. 87; E-print: nucl-th/9812038.

(30) F. X. Gallmeier and S. G. Mashnik, Proc. AccApp00, 2000, Washington DC, USA, (ANS, La Grange Park, 2001), p. 342.

(31) F. X. Gallmeier, Proc. ICRS-10/RPS 2004, 2004, Funchal, Madeira, Portugal, http://www.itn.mces.pt/ICRS-RPS/ presentations.html, in press.

(32) A. S. Iljinov, M. V. Kazarnovsky, and E. Ya. Paryev, Intermediate-Energy Nuclear Physics, (CRC Press, Boca Raton, 1994).

(33) A. S. Iljinov, I. A. Pshenichnov, N. Bianchi, E. De Sanctis, V. Muccifora, M. Mirazita, and P. Rossi, Nucl. Phys. A 616, 575 (1997)

(34) T. Gabriel, G. Maino, and S. G. Mashnik, JINR Preprint E2-94-424, Dubna, 1994; Proc. XII Int. Sem. on High 
Energy Phys. Problems, Dubna, Russia, 1994, ed. A. M. Baldin and V. V. Burov (JINR E1,2-97-79, Dubna, 1997), p. 309.

(35) F. G. Gereghi, V. A. Zolotarevsky, K. K. Gudima, S. G. Mashnik, and L. V. Bordianu, Calculation of Cross Sections for Photoneutron Reactions, Kishinev State University (1990).

(36) K. Ukai and T. Nakamura, INS-T-550 (1997), Inst. for Nucl. Study, University of Tokyo, and private communication from K. Ukai to SGM (1997).

(37) S. I. Alekhin, A. Baldini, P. Capiluppi, M. Cobal, V. V. Ezhela, V. Flaminio, G. Giacomelli, S. B. Lugovsky, G. Mandrioli, W. G. Moorhead, D. R. O. Morrison, N. Rivoire, A. M. Rossi, P. Serra, A. N. Tolstenkov, and O. P. Yushchenko, CERN-HERA 87-01, CERN, Geneva (1987).

(38) D. W. Menze, W. Pfeil, and R. Wilcke, Compilation of Pion Photoproduction Data (Physics Data 7-1, Physikalisches Institut der Universität Bonn, Geramany, 1977); Compilation of Pion Photoproduction Data, ed. W. Pfeil, R. Wilcke, and D. W. Menze (Eggenstein-Leopoldshafen Karlsruhe Fachinformationszent. Kernforsch. Zentralstelle At. Energ., 1977).

(39) H. Genzel, P. Joos, and W. Pfeil, Photoproduction of Elementary Particles, Landolt-Börnstein, New Series, Group I: Nuclear and Particle Physics, Vol. 8, ed. H. Schoper (Springer-Verlag, Heidelberg, 1973).

(40) E. Mazzucato, P. Argan, G. Audit, A. Bloch, N. de Botton, N. d'Hose, J. -L. Faure, M. L. Ghedira, C. Guerra, J. Martin, C. Schuhl, G. Tamas, and E. Vincent, Phys. Rev. Lett. 57, 3144 (1986).

(41) C. Ward, B. Kenton, and C. York, Phys. Rev. 159, 1176 (1967).

(42) H. R. Crouch, Jr., R. Hargraves, B. Kendall et al. (Cambridge Bubble Chamber Group), Phys. Rev. 155, 1477 (1967).

(43) W. J. Podilsky, Thesis, University of California, Berkeley, 1971.

(44) O. Bartholomy, V. Crede, H. van Pee, for the CB-ELSA Collaboration, E-print: hep-ex/0407022.

(45) M. Fuchs, J. Ahrens, G. Anton, R, Averbeck, R. Beck, A. M. Bernstein, A. R. Gabler, F. Härter, et al., Phys. Lett. B 368, 20 (1996).

(46) S. D. Ecklund and R. L. Walker, Phys. Rev. 159, 1195 (1967).

(47) C. Betourne, J. C. Bizot, J. Perez-Y-Jorba, and D. Treille, Phys. Lett. B 24, 590 (1967).

(48) D. A. McPherson, D. C. Gates, R. W. Kenney, and W. P. Swanson, Phys. Rev. 136, B1465 (1964).

(49) G. Fischer, G. von Holtey, G. Knop, and J. Stümpfig, Z. Phys. 253, 38 (197.2)

(50) T. Fujii, T. Kondo, F. Takasaki, S. Yamada, S Homma, K. Huke, S. Kato, H. Okuno, I. Endo, and H. Fuji, Nucl. Phys. B 120, 395 (1977).

(51) M. MacCormick, G. Audit, N. d'Hose, L. Ghedira, V. Isbert, S. Kerhoas, L. Y. Murphy, G. Tamas, P. A. Wallace, S. Altieri, A. Braghieri, P. Pedroni, T. Pinelli, J. Ahrens, R. Beck, J. R. M. Annand, R. A. Crawford, J. D. Kellie, I. J. D. MacGregor, B. Dolbilkin, and A. Zabrodin, Phys. Rev. C 53, 41 (1996).

(52)Data Files and Plots of Cross Sections and Related Quantities. Hadron(Photon)-Hadron(Photon) Collisions, Courtesy of the COMPAS Group, IHEP, Protvino, Russia, http://wwwppds.ihep.su:8001/hadron.html; http://pdg.lbl.gov/2002/contents plots.html.

(53) R. Bernabei, A. Incicchitti, M. Mattioli, P. Picozza, D. Prosperi, L. Casano, S. d'Angelo, M. P. De Pascale, C. Schaerf, G. Giordano, G. Matone, S. Frullani, and B. Girolami, Phys. Rev. Lett. 57, 1542 (1986)

(54) J. Arends, H. J. Gassen, A. Hegerath, B. Mecking, G.
Nöldeke, P. Prenzel, T. Reichelt, A. Voswinkel, and W. W. Sapp, Nucl. Phys. A 412, 509 (1984).

(55) H. O. Meyer, J. R. Hall, M. Hugi, H. J. Karwowski, R. E. Pollock, and P. Schwandt, Phys. Rev. C 31, 309 (1985).

(56) P. Levi Sandri, M. Anghinolfi, N. Bianchi, G. P. Capitani, P. Corvisiero, E. De Sanctis, C. Guaraldo, V. Lucherini, V. Muccifora, E. Polli, A. R. Reolon, G. Ricco, P. Rossi, M. Sanzone, and M. Taiuti, Phys. Rev. C 39, 1701 (1989), and compilation therein.

(57) J. M. Cameron, C. A. Davis, H. Fielding, P. Kitching, J. Pasos, J. Soukup, J. Uegaki, J. Wesick, H. S. Wilson, R. Abegg, D. A. Hutcheon, C. A. Miller, A. W. Stetz, and I. L. van Heerden, Nucl. Phys. A 458, 637 (1986).

(58) M. Hugi, J. M. Cameron, M. Ahmad, J. Collot, G. Gaillard, J. S. Wesick, G. W. R. Edwards, H. Fielding, D. A. Hutcheon, R. Abegg, C. A. Miller, P. Kitching, N. E. Davison, N. R. Stevenson, and I. J. van Heerden, Nucl. Phys. A 472, 701 (1987).

(59) P. Dougan, V. Ramsay, and W. Stiefler, Z. Phys. 280, 341 (1977).

(60) D. M. Skopik, Y. M. Shin, M. C. Phenneger, and J. J. Murphy, II, Phys. Rev. C 9, 531 (1974).

(61) R. L. Anderson, R. Prepost, and B. H. Wiik, Phys. Rev. Lett. 22, 651 (1969).

(62) J. Buon, V. Gracco, J. Lefrancois, P. Lehmann, B. Merkel, and P. Roy, Phys. Lett. B 26, 595 (1968).

(63) J. Ahrens, H. B. Eppler, H. Gimm, M. Kröning, P. Riehn, H. Wäffer, A. Zieger, and B. Ziegler, Phys. Lett. B 52, 49 (1974).

(64) E. A. Whalin, B. D. Schriever, and A. O. Hanson, Phys. Rev. 101, 377 (1956).

(65) J. C. Keck and A. V. Tollestrup, Phys. Rev. 101, 360 (1956).

(66) J. Halpern and E. V. Weinstock, Phys. Rev. 91, 934 (1953), and compilation therein.

(67)E. De Sanctis, M. Anghinolfi, G. P. Capitani, P. Corvisiero, P. Di Giacomo, C. Guaraldo, V. Lucherini, E. Polli, A. R. Reolon, G. Ricco, M. Sanzone, and A. Zucchiatti, Phys. Rev. C 34, 413 (1986), and compilation therein.

(68) EXFOR Data Base, e.g. at: http://www.nea.fr/html/dbdata/ $\mathrm{x} 4 / \mathrm{x} 4$ ret.html.

(69) K. Y. Hara, H. Utsunomiya, S. Goko, H. Akimune, T. Yamagata, M. Ohta, H. Toyokawa, K. Kubo, A. Uritani, Y. Shibata, Y.-W. Lui, and H. Ohgaki, Phys. Rev. D 68, 072001 (2003)

(70) R. Bernabei, A. Chisholm, S. d'Angelo, M. P. De Pascale, P. Picozza, C. Schaerf, P. Belli, L. Casano, A. Incicchitti, D. Prosperi, and B. Girolami, Phys. Rev. C 38, 1990 (1988).

(71) Y. Birenbaum, S. Kahane, and R. Moreh, Phys. Rev. C 32, 1825 (1985).

(72) R. Moreh, T. J. Kennett, and W. V. Prestwich, Phys. Rev. C 39, 1247 (1989)

(73) A. Braghieri, L. Y. Murphy, J. Ahrens, G. Audit, N. d'Hose, V. Isbert, S. Kerhoas, M. Mac-Cormick, P. Pedroni, T. Pinelli, G. Tamas, and A. Zabrodin, Phys. Lett. B 363, 46 (1995).

(74) K. Abe, T. C. Bacon, J. Ballam et al. (SLAC Hybrid Facility Photon Collaboration), Phys. Rev. Lett. 53, 751 (1984).

(75) A. Piaza, G. Susinno, L. Fiore, G. Gialanella, E. LodiRizzini, G. C. Mantovani, A. Piazzoli, F. Carbonara, G. Palomba-Nicodemi, and R. Rinzivillo, Lett. Nouvo Cimento 3, 403 (1970).

(76) R. Erbe et al. (ABBHHM Collaboration), Phys. Rev. 175, 1669 (1968).

(77) M. Kotulla, J. Ahrens, J. R. M. Annand, R. Beck, D. Hornidge, S. Janssen, B. Krusche, J. C. McGeorge, I. J. D. MacGregor, J. G. Messchendorp, V. Metag, R. Novotny, M. Pfeiffer, R. O. Owens, M. Rost, S. Schadmand, and D. P. Watts, Phys. Lett. B 578, 63 (2004). 
(78) M. Wolf, J. Ahrens, R. Beck, V. Hejny, J. D. Kellie, M. Kotulla, B. Krusche, V. Kuhr, R. Leukel, V. Metag, J. C. Nacher, R. Novotny, V. Olmos de León, R. O. Owens, F. Rambo, A. Schmidt, M. Schumacher, U. Siodlaczek, H. Ströher. J. Weiß, and F. Wissmann, Eur. Phys. J. A 9, 5 (2000).

(79) F. Härter, J. Ahrens, R. Beck, B. Krusche, V. Metag, M. Schmitz, H. Ströher, Th. Walcher, and M. Wolf, Phys. Lett. B 401, 229 (1997).

(80) J. Ahrens et al. (GDH and A2 Collaboration), Phys. Lett. B 551, 49 (2003).

(81) W. Langgärtner, J. Ahrens, R. Beck, V. Hejny, M. Kotulla, B. Krusche, V. Kuhr, R. Leukel, J. D. McGregor, J. G. Messchendorp, V. Metag, R. Novotny, V. Olmos de León, R. O. Owens, F. Rambo, S. Schadmand, A. Schmidt, U. Siodlaczek, H. Ströher, J. Weiß, F. Wissmann, and M. Wolf, Phys. Rev. Lett. 87, 052001 (2001).

(82) K.-H. Schmidt, personal Web page: http://www-wnt. gsi.de/kschmidt/.

(83) R. W. Clifft, E. Gabathuler, L. S. Littenberg, R. Marshall, S. E. Rock, J. C. Thompson, D. L. Ward, and G. R. Brookes, Phys. Rev. Lett. 33, 1500 (1974).

(84) H. Genzel, E. Hilger, G. Knop, H. Kemen, and R. Wedemeyer, Z. Phys. 268, 43 (1974).

(85) G. Fischer, H. Fischer, G. von Holtey, H. Kämpgen, G. Knop, P. Schulz, H. Wessels, W. Braun-schweig, H. Genzel, and R. Wedemeyer, Nucl. Phys. B 16, 93 (1970).

(86) W. S. McDonald, V. Z. Peterson, and D. R. Corson, Phys. Rev. 107, 577 (1957).

(87) Yu. A. Alexandrov, V. A. Kozlov, and V. V. Pavlovskaya, Yad. Fiz. 28, 670 (1978).

(88) R. L. Walker, D. C. Oakley, and A. V. Tollestrup, Phys. Rev. 97, 1279 (1955).

(89) Yoshioka 1977 INS-281 data tabulated at: http://wwwspires.dur.ac.uk/hepdata/reac2.html.

(90) H. Becks, P. Feller, D. Menze, U. Opara, W. Schultz, and W. J. Schwille, Nucl. Phys. B 60, 267 (1973).

(91) S. Almehed, G. von Dardel, G. Jarlskog, L. Jönsson, B. Lörstad, J. Palmer, R. Peterson, and L. Svensson, Physica Scripta 13, 321 (1976).

(92) H. De Staebler, Jr., E. F. Erickson, A. C. Hearn, and C. Schaere, Phys. Rev. 140, B336 (1965).

(93) P. Feller, H. Herr, E. Hilger, V. Kadansky, D. Menze, Th. Miczaika, U. Opra, and W. J. Schwille, Phys. Lett. B 49, 197 (1974).

(94) Wolverton 1968 data tabulated at: http://www-spires.dur. ac.uk/hepdata/reac2.html.

(95) Wermes 1978 data tabulated at: http://www-spires.dur. ac.uk/hepdata/reac2.html.

(96) B. Delcourt, J. Lefrançois, G. Parrour, J. P. Perez-YJorba, and G. Sauvage, Phys. Lett. B 29, 70 (1960).

(97) R. M. Worlock, Phys. Rev. 117, 537 (1960).

(98) D. Husmann, W. Jansen, B. Löhr, and H. Schilling, Nucl. Phys. B 126, 436 (1977).

(99) J. S. Barton, P. S. L. Booth, L. J. Carroll, J. R. Holt, J. N. Jackson, G. Moscatti, and J. R. Wormald, Nucl. Phys. B 84, 449 (1975).

(100) H. E. Jackson, J. W. DeWire, and R. M. Littauer, Phys. Rev. 119, 1381 (1960).

(101) K. H. Althoff, M. Gies, O. Kaul, K. Königsmann, D. Menze, W. Meyer, T. Miczaika, E. Roder-burg, and W. Schwille, Z. Phys. C 1, 257 (1979).

(102)Lohr 1970 data tabulated at: http://www-spires.dur.ac.uk/ hepdata/reac2.html.

(103) M. I. Adamovich, V. G. Larionova, R. A. Latypova, A. I. Lebedev, S. P. Kharlamov, and F. R. Yagudina, Zh. Exp. Teor. Fiz. 53, 528 (1967).

(104) M. I. Adamovich, E. G. Gorzhevskaya, V. G. Larionova, V. M. Popova, S. P. Kharlamov, and F. R. Yagudina, Zh.
Exp. Teor. Fiz. 11, 1078 (1960).

(105) K. Althoff, Z. Phys. 175, 34 (1963).

(106) D. Freytag, W. J. Schwille, and R. J. Wedemeyer, Z. Phys. 186, 1 (1965).

(107) M. Beneventano, G. Bernardini, D. Carlson-Lee, G. Stoppini, and L. Tau, Nouvo Cimento 4, 323 (1956).

(108) R. L. Walker, J. G. Teasdale, V. Z. Peterson, and J. I. Vette, Phys. Rev. 99, 210 (1955).

(109) T. Fujii, T. Kondo, F. Takasaki, S. Yamada, S. Homma, K. Huke, S. Kato, H. Okuno, I. Endo, and H. Fujii, Nucl. Phys. B 120, 395 (1977).

(110) G. Fischer, G. von Holtey, G. Knop, and J. Stumpfig, Z. Phys. 253, 38 (1972); G. Fischer, H. Gischer, M. Heuel, G. von Holtey, G. Knop, and J. Stumpfig, Nucl. Phys. B 16, 119 (1970).

(111)C. Betourne, J. C. Bizot, J. Perez-Y-Jorba, and D. Treille, Phys. Rev. 172, 1343 (1968).

(112) M. Beneventano, R. Finzi, L. Mezzetti, L. Paoluzi, and S. Tazzari, Nuovo Cimento 28, 1464 (1963).

(113)F. P. Dixon and R. L. Walker, Phys. Rev. Lett. 1, 458 (1958).

(114)H.-W. Dannhausen, G. Fischer, H. M. Fischer, K. Kircher, M. Leneke, W. Niehaus, and H. Wessels, Nucl. Phys. B 61, 285 (1973).

(115) T. Fujii, H. Okuno, S. Orito, H. Sasaki, T. Nozaki, F. Takasaki, K. Takikawa, K. Amako, I. Endo, K. Yoshida, M. Higuchi, M. Sato, and Y. Sumi, Phys. Rev. Lett. 26, 1672 (1971).

(116) K. H. Althoff, G. Anton, B. Bock, D. Bour, W. Ferber, W. Ferber, H. W. Gelhausen, T. Jahnen, O. Kaul, D. Menze, W. Meyer, T. Miczaika, R. Müller, E. Roderburg, W. Ruhm, E. Schilling, W. J. Schwille, D. Sundermann, and K. Wagener, Z. Phys. C 18, 199 (1983).

(117) S. D. Ecklund and R. L. Walker, Phys. Rev. 159, 1195 (1967).

(118) R. A. Alvarez, G. Cooperstein, K. Kalata, R. C. Lanza, and D. Luckey, Phys. Rev. D 1, 1946 (1970); Phys. Rev. Lett. 21, 1019 (1968).

(119) G. Buschhorn, J. Carroll, R. D. Eandi, P. Heide, R. Hübner, W. Kern, U. Kötz, P. Schmüser, and H. J. Skronn, Phys. Rev. Lett. 18, 571 (1967).

(120) L. Y. Zhu, J. Arrington, T. Averett et al., Phys. Rev. Lett. 91, 022003 (2003).

(121) K. Ekstrand, A. Browman, L. Hand, and M. E. Nordberg, Jr., Phys. Rev. D 6, 1 (1972).

(122) V. K. Luk'yanov, V. A. Seliversov, and V. D. Toneev, Yad. Fiz. 21, 992 (1975).

(123) K. K. Gudima, G. A. Ososkov, and V. D. Toneev, Yad. Fiz. 21, 260 (1975).

(124) S. G. Mashnik and V. D. Toneev, JINR Communication P4-8417, Dubna (1974).

(125) A. G. Belov, Yu. P. Gangrskii, K. K. Gudima, and P. Zuzaan, At. Energ. 88, 391 (2000).

(126) M. B. Chadwick, P. Oblizinsky, A. I. Blokhin, T. Fukahori, Y. Han, Y.-O. Lee, M. N. Martins, S. F. Mughabghab, V. V. Varlamov, B. Yu, and J. Zhang, IAEA-TECDOC-1178, IAEA, Vienna, (2000); Los Alamos National Laboratory Report LA-UR-02-2002 (2002).

(127) J. Ahrens, Nucl. Phys. A 446, 229c (1985).

(128) G. R. Brookes, A. S. Clough, J. H. Freeland, W. Galbraith, A. F. King, T. A. Armstrong, W. R. Hogg, G. M. Lewis, A. W. Robertson, W. R. Rawlinson, N. R. S. Tait, J. C. Thompson, and D. W. L. Tolfree, Phys. Rev. D 8, 2826 (1973).

(129) N. Bianchi, V. Muccifora, E. De Sanctis, A. Fantoni, P. Levi Sandri, E. Polli, A. R. Reolon, P. Rossi, M. Anghinolfi, P. Corvisiero, M. Ripani, M. Sanzone, M. Taiuti, and A. Zucchiatti, Phys. Rev. C 54, 1688 (1996). 
(130) N. A. Burgov, G. V. Danilyan, B. S. Dolbilkin, L. E. Lazareva, and F. A. Nikolaev, Zh. Eksp. Teor. Fiz. 45, 1694 (1963).

(131)E. A. Arakelyan, G. L. Bayatyan, G. S. Vartanyan, N. K. Grigoryan, A. O. Kechechyan, S. G. Knyazyan, A. T. Margaryan, G. G. Marikyan, S. S. Stepanyan, and S. R. Shakhazizyan, Yad. Fiz. 38, 980 (1983).

(132) E. A. Arakelyan, G. L. Bayatyan, G. S. Vartanyan, A. R. Voskanyan, N. K. Grigoryan, S. G. Knyazyan, A. T. Margaryan, G. G. Marikyan, S. S. Stepanyan, E. M. Oganesyan, and S. R. Shakhazizyan, Yad. Fiz. 42, 3 (1985).

(133) V. Muccifora, N. Bianchi, A. Deppman, E. De Sanctis, M. Mirazita, E. Polli, and P. Rossi, Phys. Rev. C 60, 064616 (1999).

(134) J. M. Wyckoff, B. Ziegler, H. W. Koch, and R. Uhlig, Phys. Rev. 137, B576 (1965).

(135) J. Ahrens, H. Borchert, K. H. Czock, H. B. Eppler, H. Gimm, H. Gundrum, M. Kröning, P. Riehn, G. Sita Ram, A. Zieger, and B. Ziegler, Nucl. Phys. A 251, 479 (1975).

(136) J. Arends, J. Eyink, A. Hegerath, K. G. Hilger, B. Mecking, G. Nöldeke, and H. Rost, Phys. Lett. B 98, 423 (1981).

(137) S. Michalowski, D. Andrews, J. Eickmeyer, T. Gentile, N. Mistry, R. Talman, and K. Ueno, Phys. Rev. Lett. 39, 737 (1977).

(138) E. A. Arakelyan, G. L. Bayatyan, G. S. Vartanyan, N. K. Grigoryan, S. G. Knyazyan, A. T. Margaryan, S. S. Stepanyan, P. K. Kir'yanov, V. A. Maisheev, and A. M. Frolov, Phys. Lett. B 79, 143 (1978).

(139) D. O. Caldwell, V. B. Elings, W. P. Hesse, R. J. Morrison, F. V. Murphy, and D. E. Young, Phys. Rev. D 7, 1362 (1973).

(140) A. Leprêtre, H. Beil, R. Bergère, P. Carlos, J. Fagot, A. De Miniac, and A. Veyssière, Nucl. Phys. A 367, 237 (1981).

(141) S. C. Fultz, B. L. Berman, J. T. Caldwell, R. L. Bramblett, and M. A. Kelly, Phys. Rev. 186, 1255 (1969).

(142) G. M. Gurevich, L. E. Lazareva, V. M. Mazur, S. Yu. Merkulov, G. V. Solodukhov, and V. A. Tyutin, Nucl. Phys. A 351, 257 (1981).

(143)O. A. P. Tavares, J. B. Martins, E. de Paiva, E. L. Moreira, J. L. Vieira, M. L. Terranova, M. Capogni, L. Casano, A. D'Angelo, D. Moricciani, F. Ghio, B. Girolami, and B. Babusci, J. Phys. G 19, 805 (1993).

(144) M. L. Terranova, O. A. P. Tavares, G. Ya. Kezerashvili, V. A. Kiselev, A. M. Milov, N. Yu. Muchnoi, A. I. Naumenkov, V. V. Petrov, I. Ya. Protopopov, E. A. Simonov, E. De Paiva, and E. L. Moreira, J. Phys. G 22, 511 (1996).

(145) M. L. Terranova, G. Ya. Kezerashvili, A. M. Milov, S. I. Mishnev, N. Yu. Muchnoi, A. I. Naumenkov, I. Ya. Protopopov, E. A. Simonov; D. N. Shatilov, O. A. P. Tavares, E. De Paiva, and E. L. Moreira, J. Phys. G 24, 205 (1998).

(146) J. B. Martins, E. L. Moreira, O. A. P. Tavares, J. L. Vieira, J. D. Pinheiro Filho, R. Bernabei, S. D'Angelo, M. P. de Pascale, C. Schaerf, and B. Girolami, Nuovo Cimento A 101, 789 (1989).

(147) J. B. Martins, E. L. Moreira, O. A. P. Tavares, J. L. Vieira, L. Casano, A. D'Angelo, C. Schaerf, M. L. Terranova, D. Babusci, and B. Girolami, Phys. Rev. C 44, 354 (1991).

(148)C. Chollet, J. Arends, H. Beil, R. Bergère, P. Bourgeois, P. Carlos, J. L. Fallou, J. Fagot, P. Garganne, A. Leprêtre, and A. Veyssière, Phys. Lett. B 127, 331 (1983).

(149) G. M. Gurevich, L. E. Lazareva, V. M. Mazur, G. V. Solodukhov, and B. A. Tulupov, Nucl. Phys. A 273, 326 (1976).

(150) Y. Birenbaum, R. Alarcon, S. D. Hoblit, R. M. Laszewski, and A. M. Nathan, Phys. Rev. C 36, 1293 (1987):

(151) G. M. Gurevich, L. E. Lazareva, V. M. Mazur, and G. V. Solodukhov, Pis'ma Zh. Eksp. Teor. Fiz. 20, 741 (1974).

(152) N. K. Sherman, W. F. Davidson, A. Nowak, M. Kosaki, J. Roy, W. Delbianco, and G. Kajrys, Phys. Rev. Lett. 54, 1649 (1985).

(153)O. A. P. Tavares, M. L. Terranova, L. Casano, A. D'Angelo, D. Moricciani, C. Schaerf, D. Babusci, B. Girolami, J. B. Martins, E. L. Moreira, and J. L. Vieira Phys. Rev. C 44, 1683 (1991).

(154) E. A. Arakelyan, A. R. Bagdsaryan, G. L. Bayatyan, G. S. Vartanyan, A. R. Voskanyan, N. KI. Grigoryan, S. G. Knyazyan, A. T. Margaryan, G. G. Marikyan, and A. K. Papyan, Yad. Fiz. 52, 1387 (1990).

(155) N. Bianchi, A. Deppman, E. De Sanctis, A. Fantoni, P. Levi Sandri, V. Lucherini, V. Muccifora, M. Anghinolfi, P. Corvisiero, G. Gervino, L. Mazzaschi, V. Mokeev, G. Ricco, M. Ripani, M. Sanzone, M. Taiuti, A. Zucchiatti, R. Bergère, P. Carlos, P. Garganne, and A. Leprêtre, Phys. Lett. B 299, 219 (1993).

(156) GEANT4 Physics Reference Manual, http://wwwasd.web. cern.ch/wwwasd/geant4/G4UsersDocuments/Overview/ $\mathrm{html} /$ index.html.

(157) J. P. Wellisch, M. Kossov, and P. Degtyarenko, E-print: nucl-th/0306012.

(158) A. V. Prokofiev, S. G. Mashnik, and A. J. Sierk, Nucl. Sci. Eng. 131, 78 (1999); E-print: nucl-th/9802027.

(159) V. V. Varlamov, N. G. Efimkin, V. V. Surgutanov, A. A. Khoronenko, and A. P. Chernyaev, CDFE/FIS2, Moscow State University (1987).

(160) V. Lucherini, C. Guaraldo, E. De Sanctis, P. Levi Sandri, E. Poli, A. R. Reolon, A. S. Iljinov, S. Lo Nigro, S. Aiello, V. Bellini, V. Emma, C. Milone, G. S. Pappalardo, and M. V. Mebel, Phys. Rev. C 39, 911 (1989).

(161) G. Andersson, I. Blomqvist, B. Forkman, G. G. Jonsson, A. Järund, K. Kroon, K. Lindgren, B. Schroder, and K. Tesh, Nucl. Phys. A 197, 44 (1972).

(162) M. L. Terranova, A. D’Angelo, J.D. Pinheiro, E. S. De Alme, E. Z. Bilbao, and J. B. Martins, Nuovo Cimento A 105, 197 (1992).

(163) C. Cetina, P. Heimberg, B. L. Berman, W. J. Briscoe, G. Feldman, L. Y. Murthy, Hall Crannell, A. Longhi, D. I. Sober, J. C. Sanabria, and G. Ya: Kezerashvili, Phys. Rev. C 65, 044622 (2002).

(164) H.-D. Lemke, B. Ziegler, M. Mutterer, J. P. Theobald, N. Cârjan, Nucl. Phys. A 342, 37 (1980).

(165) V. Bellini, V. Emma, S. Lo Nigro, C. Milone, G. S. Pappalardo, E. De Sanctis, P. Di Giacomo, C. Guaraldo, V. Lucherini, E. Polli, and A. R. Reolon, Lett. Nouvo Cimento 36, 587 (1983).

(166) L. G. Moretto, R. C. Gatti, S. G. Thompson, J. T. Routti, J. H. Heisenberg, L. M. Middleman, M. R. Yearian, and R. Hofstadter, Phys. Rev. 179, 1176 (1969).

(167) E. V. Minarik and V. A. Novikov, Zh. Eksp. Teor. Fiz. 32, 241 (1957).

(168) M. L. Terranova, G. Ya. Kezerashvili, V. A. Kiselev, A. M. Milov, S. I. Mishnev, I. Ya. Protopopov, V. N. Rotaev, D. N. Shatilov, and O. A. P. Tavares, J. Phys. G 22, 1661 (1996).

(169) Yu. N. Ranyuk and P. V. Sorokin, Yad. Fiz. 5, 37 (1967).

(170) C. Guaraldo, V. Lucherini, E. De Sanctis, P. Levi Sandri, E. Polli, A. R. Reolon, S. Lo Nigro, S. Aiello, V. Bellini, V. Emma, C. Milone, and G. S. Pappalardo, Phys. Rev. C 36, 1027 (1987).

(171) J. A. Jungerman and H. M. Steiner, Phys. Rev. 106, 585 (1957).

(172) J. T. Caldwell, E. J. Dowdy, B. L. Berman, R. A. Alvarez, and P. Meyer, Phys. Rev. C 21, 1215 (1980).

(173) S. P. Kapitza, N. S. Rabotnov, G. N. Smirenkin, A. S. 
Soldatov, L. N. Usachev, and Yu. M. Tsipenyuk, Pis'ma Zh. Eksp. Teor. Fiz. 9, 128 (1969).

(174) A. Leprêtre, R. Bergère, P. Bourgeois, P. Calos, J. Fagot, J. L. Fallou, P. Garganne, A. Veyssière, H. Ries, R. Göbel, U. Kneissl, G. Mank, H. Ströher, W. Wilke, D. Ryckbosch, and J. Jury, Nucl. Phys. A 472, 533 (1987).

(175) A. Veyssière, H. Beil, R. Bergère, P. Carlos, A. Lepretre, Nucl. Phys. A 199, 45 (1973).

(176) H. X. Zhang, T. R. Yeh, and H. Lancman, Phys. Rev. C 34, 1397 (1986).

(177) B. L. Berman, J. T. Caldwell, E. J. Dowdy, F. S. Dietrich, P. Meyer, and R. A. Alvarez, Phys. Rev. C 34, 2201 (1986).

(178) M. A. P. V. de Moraes and M. F. Cesar, Nuovo Cimento A106, 1165 (1993).

(179) J. R. Huizenga, K. M. Clarke, J. E. Gindler, and R. Vandenbosch, Nucl. Phys. 34, 439 (1962).

(180) Yu. B. Ostapenko, G. N. Smirenkin, A. S. Soldatov, V. E. Zhuchko, and Yu. M. Tsipenyuk, VANT, Seriya Yadernye Konstanty 3(30), 3 (1978).

(181)H. Ries, U. Kneissl, G. Mank, H. Ströher, W. Wilke, R. Bergère, P. Bourgeois, P. Carlos, J. L. Fallou, P. Garganne, A. Veyssière, and L. S. Cardman, Phys. Lett. B 139, 254 (1984).

(182) Th. Frommhold, F. Steiper, W. Henkel, U. Kneissl, J. Ahrens, R. Beck, J. Peise, M. Schmitz, I. Anthony, J. D. Kellie, S. J. Hall, and G. J. Miller, Z. Phys. A 350, 249 (1994).

(183) W. Cesar Khouri, Final Report to the IAEA on the Research Contract NDT-18 (1983).

(184) A. S. Soldatov, VANT, Seriya Yadernye Konstanty 1-2, 8 (1997).

(185) S. G. Mashnik, K. K. Gudima, I. V. Moskalenko, R. E. Prael, and A. J. Sierk, Adv. Space Res. 34, 1288 (2004).

(186) V. D. Toneev and K. K. Gudima, Nucl. Phys. A 400, $173 \mathrm{c}(1983)$.

(187) R. A. Schumacher, G. S. Adams, D. R. Ingham, J. L. Matthews, W. W. Sapp, R. S. Turley, R. O. Owens, and B. L. Roberts, Phys. Rev. C 25, 2269 (1982).

(188) M. Anghinolfi, V. Lucherini, N. Bianchi, G. P. Capitani, P. Corvisiero, E. De Sanctis, P. Di Giacomo, C. Guaraldo, P. Levi-Sandri, E. Polli, A. R. Reolon, G. Ricco, M. Sanzone, and M. Taiuti, Nucl. Phys. A 457, 645 (1986).

(189) K. G. Fissum, H. S. Caplan, E. L. Kallin, D. M. Skopik, J. M. Vogt, M. Frodyma, D. P. Rosenzweig, D. W. Storm, G. V. O`Rielly, and K. R. Garrow, Phys. Rev. C 53, 1278 (1996).

(190) L. I. Schiff, Phys. Rev. 83, 252 (1951).

(191)D. N. Olson, Ph.D. thesis, Cornell University, 1960.

(192) D. H. Boal and R. M. Woloshin, Phys. Rev. C 23, 1206 (1981).

(193) J. L. Matthews and W. Turchinetz, LNS Internal Report No. 110 (1966).

(194) S. Kabe, S. Kato, T. Kifune, Y. Kimura, M. Kobayashi, K. Kondo, Y. Nagashima, and T. Nishikawa, J. Phys. Soc. Jpn. 19, 1800 (1964).

(195) K. Sakamoto, J. Nucl. Radiochem. Sci. 4, A9 (2003).

(196) H. Haba, J. Nucl. Radiochem. Sci. 3, A11 (2002).

(197) T. A. Gabriel and R. G. Alsmiller, Jr., Phys. Rev. 182, 1035 (1969).

(198) T. A. Gabriel, M. P. Guthrie, and O. W. Hermann, Oak
Ridge Narional Laboratory Report ORNL-4687 (1971).

(199) C. Y. Fu, T. A. Gabriel, and R. A. Lille, Proc. SATIF-3, Sendai, Japan, 1997, (NEA/OECD, Paris, France, 1998), p. 49.

(200) T. Sato, K. Shin, S. Ban, Y. Namito, H. Nakamura, and H. Hirayama, Nucl. Instrum. Meth. A 437, 471 (1999).

(201) T. Sato, K. Shin, S. Ban, T. A. Gabriel, C. Y. Fu, and H. S. Lee, Proc. MC2000, Lisbon, Portugal, 2000, ed. A. Kling, F. J. C. Barão, M. Nakagawa, L. Távora, and P. Vaz, Springer, p. 1139 (Berlin, 2001).

(202) H. Haba, M. Igarashi, K. Washiyama, H. Matsumura, M. Yamashita, K. Sakamoto, Y. Oura, S. Shibata, M. Furukawa, and I. Fujiwara, J. Nucl. Radiochem. Sci. 1, 53 (2000).

(203) H. Haba, M. Kasaoka, M. Igarashi, K. Washiyama, H. Matsumura, Y. Oura, S. Shibata, K. Sakamoto, M. Furukawa, and I. Fujiwara, Radiochim. Acta 90, 371 (2002).

(204) T. Methasiri and S. A. E. Johansson, Nucl. Phys. A 167, 97 (1971).

(205) I. Kroon and B. Forkman, Nucl. Phys. A 179, 141 (1972).

(206) P. David, J. Debrus, U. Kim, G. Kumbartzki, H. Mommsen, W. Soyez, K. H. Speidel, and G. Stein, Nucl. Phys. A 197, 163 (1972).

(207) G. A. Vartapetyan, N. A. Demekhina, V. I. Kasilov, Yu. N. Ranyuk, P. V. Sorokin, and A. G. Khudaverdyan, Yad. Fiz. 14, 65 (1971).

(208) V. Emma, S. Lo Nigro, and C. Milone, Nucl. Phys. A 257, 438 (1976).

(209)F. M. Kiely and B. D. Pate, Z. Phys. A 279, 331 (1976).

(210) G. Bernardini, R. Reitz, and E. Segre, Phys. Rev. 90, 573 (1953).

(211) H. G. de Carvalho, G. Cortini, E. del Giudice, G. Potenza, R. Rinzivillo, and G. Ghigo, Nuovo Cimento 32, 293 (1964).

(212) H. G. de Carvalho, J. B. Martins, O. A. Tavares, V. di Napoli, M. L. Teranova, and K. Tesch, Lett. Nuovo Cimento 14, 615 (1975).

(213) K. Sakamoto, S. R. Sarkar, Y. Oura, H. Haba, H. Matsumura, Y. Miyamoto, S. Shibata, M. Furukawa, and I. Fujiwara, Phys. Rev. C 59, 1497 (1999).

(214) S. R: Sarkar, M. Soto, Y. Kubota, M. Yoshida, T. Fukasawa, K. Matsumoto, K. Kawaguchi, K. Sakamoto, S. Shibata, M. Furukawa, and I. Fujiwara, Radiochim. Acta 55, 113 (1991).

(215) H. Matsumura, K. Washiyama, H. Haba, Y. Miyamoto, Y. Oura, K. Sakamoto, S. Shibata, M. Furukawa, I. Fujiwara, H. Nagai, T. Kobayashi, and K. Kobayashi, Radiochim. Acta 88, 313 (2000).

(216) M. Yamashita, K. Yoshida, Y. Terada, A. Nagano, Y. Kawashima, D. Osada, H. Matsumura, K. Washiyama, K. Sakamoto, Y. Miyamoto, Y. Oura, S. Shibata, I. Fujiwara, and M. Furukawa, Suppl. J. Nucl. Radiochem. Sci. 1, 3P17 (1999).

(217) Yu. E. Titarenko, O. V. Shvedov, V. F. Batyaev, E. I. Karpikhin, V. M. Zhivun, A. B. Koldobsky, R. D. Mulambetov, S. V. Kvasova, A. N. Sosnin, S. G. Mashnik, R. E. Prael, A. J. Sierk, T. A. Gabriel, M. Saito, and H. Yasuda, Phys. Rev. C 65, 064610 (2002).

(218) D. Ryckbosch, L. Van Hoorebeke, R. Van de Vyver, F. De Smet, J-O. Adler, D. Nilsson, B. Schröder, and R. Zorro, Phys. Rev. C 42, 444 (1990). 